\title{
Multiobjective Optimization of a Fractional-Order PID Controller for Pumped Turbine Governing System Using an Improved NSGA-III Algorithm under Multiworking Conditions
}

\author{
Chu Zhang, ${ }^{1,2}$ Tian Peng $\left(\mathbb{D},{ }^{1}\right.$ Chaoshun Li $\left(\mathbb{D},{ }^{2}\right.$ Wenlong Fu $\mathbb{D}{ }^{3}$ \\ Xin Xia, ${ }^{1}$ and Xiaoming Xue ${ }^{1}$ \\ ${ }^{1}$ College of Automation, Huaiyin Institute of Technology, Huaian 223003, China \\ ${ }^{2}$ School of Hydropower and Information Engineering, Huazhong University of Science and Technology, Wuhan 430074, China \\ ${ }^{3}$ College of Electrical Engineering \& New Energy, China Three Gorges University, Yichang 443002, China
}

Correspondence should be addressed to Tian Peng; husthydropt@126.com and Chaoshun Li; csli@hust.edu.cn

Received 1 November 2018; Revised 19 December 2018; Accepted 6 February 2019; Published 27 February 2019

Guest Editor: Izaskun Garrido

Copyright (C) 2019 Chu Zhang et al. This is an open access article distributed under the Creative Commons Attribution License, which permits unrestricted use, distribution, and reproduction in any medium, provided the original work is properly cited.

In order to make the pump turbine governing system (PTGS) adaptable to the change of working conditions and suppress the frequency oscillation caused by the "S" characteristic area running at middle or low working water heads, the traditional singleobjective optimization for fractional-order PID (FOPID) controller under single working conditions is extended to a multiobjective framework in this study. To establish the multiobjective FOPID controller optimization (MO-FOPID) problem under multiworking conditions, the integral of the time multiplied absolute error (ITAE) index of PTGS running at low and high working water heads is adopted as objective functions. An improved nondominated sorting genetic algorithm III based on Latin hypercube sampling and chaos theory (LCNSGA-III) is proposed to solve the optimization problem. The Latin hypercube sampling is adopted to generate well-distributed initial population and take full of the feasible domain while the chaos theory is introduced to enhance the global search and local exploration ability of the NSGA-III algorithm. The experimental results on eight test functions and a real-world PTGS have shown that the proposed multiobjective framework can improve the Pumped storage units' adaptability to changeable working conditions and the proposed LCNSGA-III algorithm is able to solve the MO-FOPID problem effectively.

\section{Introduction}

With the continuous expansion of the power system scale, the power grid's requirements for power quality, safety, and intelligence have been constantly improving. Pumped storage units (PSUs) have played an important role in maintaining the balance of power supply and demand because of their fast start-up and shutdown speed, flexible working condition conversion, excellent peak-load regulation, and frequency regulation ability [1-3]. Pump turbine governing system is the core control system of the pumped storage power station which is responsible for stabilizing the unit frequency and regulating the unit power $[4,5]$. Due to the huge flow inertia of the long-distance water pipeline and the existence of the unstable "S" characteristic area, the optimal control of PTGS is highly complex [6]. Therefore, it is of great theoretical value and practical significance to explore optimization methods for PTGS and research new control laws. The control quality of PSU and the dynamic response performance of PTGS can then be improved.

The classical Proportional-Integral-Derivative (PID) controller is widely used in the optimal control of PTGS because of its simple and reliable structure and easy adjustment of control parameters [7-10]. However, due to the strong nonlinear characteristics of different parts of PSU and the changeable working conditions, the traditional PID controller often 
fails to realize the global optimization of PTGS. The control problems of phase modulation instability and no-load frequency fluctuation are becoming increasingly prominent. As an extension of the classical PID controller, the fractionalorder PID controller (FOPID) has attracted the attention of many scholars for its better adaptability and flexibility and greater potential to obtain better control performance [1115]. Li et al. [16] proposed an improved gravitational search algorithm using the Cauchy and Gaussian mutations to adjust the parameters of the FOPID controller automatically. A number of tests have shown that the FOPID controller can improve the dynamic characteristics and stability of regulation frequency of PSUs. In Xu et al. [17], a robust nonfragile FOPID controller was proposed for PTGS. The parameters of the FOPID controller were selected using the bacterial foraging algorithm and multiscenario analysis functions. The FOPID controller turned out to have obtained higher robustness and stability compared with the traditional PID controller. And it can fully track the nonlinear characteristics of PTGS in the "S" area. Xu et al. [18] proposed an adaptively fast fuzzy fractional-order PID (AFFFOPID) control method for PSUs by combining a fuzzy fractional-order PD controller with a fuzzy fractional-order PI controller. Experiments of PTGS at various water heads under unload running condition have shown that the controller can effectively improve the performance and control quality of PSUs during the transient process.

Apart from the advantages of the FOPID compared with the PID controller, one of the most important and challenge issues of the employment of FOPID controller in PTGS is the optimal optimization of its parameters. With the continuous development of optimization algorithms and control theory in recent years, scholars have combined the FOPID controller with intelligent algorithms to achieve the optimal tuning of control parameters and improvement of control laws for PTGS [19]. The related works on optimal optimization for PID controller or hydroturbine governing system (HTGS) can also provide meaningful reference for the research of FOPID controller for PTGS. Fang et al. [20] developed an improved particle swarm optimization (PSO) algorithm for optimal tuning of PID control parameters for water turbine governor. Simulation results have demonstrated the stable convergence characteristic and good computational ability of the developed optimization stagey. Kou et al. [21] proposed a novel BFO-PSO algorithm by introducing PSO into the framework of the bacterial foraging optimization (BFO) algorithm to improve the control performance of the PID controller for HTGS. The advantages of the proposed algorithm to the BFO and PSO algorithms have been demonstrated through experiments at real working conditions. Wang et al. [22] proposed a three-stage start-up strategy for PSUs by opening guide vanes to a large opening degree firstly and then reducing the opening degree and finally switching to the PID controller. The switching time and PID control parameters are optimized synchronously using an integrated optimization scheme based on artificial sheep algorithm (ASA). Simulation results under various water heads have shown that the control strategy can shorten the start-up time and reduce the speed oscillation.
Some researchers have paid attention to multiobjective designing to consider multiobjectives that reflect the specific characteristics of the control system [23, 24]. Zamani et al. [25] developed a multiobjective cuckoo search approach to optimize the parameters of a FOPID controller. Sánchez et al. [26] proposed a multiobjective optimization strategy for identifying the optimal solution for a robust FOPID controller. Zhao et al. [27] proposed a parameter tuning scheme based on two lbests multiobjective PSO (2LB-MOPSO) to minimize the integral squared error and balanced robust performance criteria of a robust PID controller simultaneously. Chen et al. [28, 29] put forward a parameter tuning scheme to optimize the integral of the squared error (ISE) and the integral of the time multiplied squared error (ITSE) performance indices of PTGS simultaneously. The adaptive grid PSO (AGPSO) and the chaotic nondominated sorting genetic algorithm II (NSGA II) were adopted to realize the optimal control of the PID and the FOPID controllers, respectively. The proposed multiobjective PID and FOPID controller turned out to have achieved better control effects than the compared methods.

It is noticed that most of the research works on optimal control of the PID or FOPID controllers are designed under a single working condition. The traditional multiobjective optimization control usually adopts a set of objective functions to obtain the Pareto optimal solutions for a certain condition and then select the compromise optimal solution. The traditional multiobjective optimization control is essentially an optimization scheme for single working conditions. However, the characteristics of the controlled object of PTGS are not only related to the nonlinear characteristics of the pump turbine, the penstock system, the generator, and the other parts of PTGS but also vary with the changes of the working conditions. The optimal control of a single working condition is often at the expense of the deterioration of some other working conditions. The optimal control of PTGS under multiworking conditions should be a process of overall tradeoff and cannot be limited to the pursuit of the optimal control under a certain working condition. In order to enhance the adaptability of PTGS to the change of working conditions, a multiobjective optimization framework which takes into account the integral of the time multiplied absolute error (ITAE) index of PTGS running at multiworking conditions is constructed. The nondominated sorting genetic algorithm III based on the Latin hypercube sampling and chaos theory (LCNSGA-III) is proposed to optimize the control parameters of the FOPID controller for PTGS under multiworking conditions.

The rest of this paper is arranged as follows: Section 2 gives a brief introduction to the multiobjective optimization problem; Section 3 builds the mathematical model of MO-FOPID under multiworking conditions; Section 4 proposes the LCNSGA-III algorithm based on Latin hypercube sampling and the chaos theory to solve the MOFOPID problem; Section 5 employs eight benchmark functions and a real-world PTGS to verify the effectiveness of the LCNSGA-III algorithm and the developed multiobjective optimization framework; Section 6 gives the conclusions. 


\section{Multiobjective Optimization Problem (MOOP)}

The purpose of the single-objective (SO) optimization problem is to obtain the optimal solution by searching for the minimum or maximum value of one single objective function. However, optimization problems in scientific research or engineering application usually contain not only one objective. These objectives are sometimes in concordance with each other, but sometimes there are conflicts between them. The objective of the multiobjective optimization problem (MOOP) is to search for the optimal solutions of all objectives, which is also known as the Pareto optimal solution [30]. A typical MOOP with $D$ decision variables, $N$ objective functions, and $m+k$ constraints can be described as follows:

$$
\begin{aligned}
& \min \quad F(X)=\left(\min f_{1}(x), \min f_{2}(x), \ldots, \min f_{i}(x),\right. \\
& \left.\ldots, \min f_{N}(x)\right) \\
& \text { st. } \quad\left\{\begin{array}{l}
g_{i}(X) \geq 0, \quad i=1,2, \ldots, m \\
h_{j}(X)=0, \quad i=1,2, \ldots, k
\end{array}\right. \\
& X=\left[x_{1}, x_{2}, \ldots, x_{d}, \ldots, x_{D},\right] \\
& x_{d \_ \text {min }} \leq x_{d} \leq x_{d \_ \text {max }} \quad d=1,2, \ldots, D
\end{aligned}
$$

where $X$ is the decision variable in $D$ dimensions; $F(X)$ is the objective function in $N$ dimensions; $g_{i}(X)$ represents the $i$ th inequality equation; $h_{j}(X)$ represents the $i$ th equality equation; $x_{d \_ \text {min }}$ and $x_{d \_ \text {min }}$ represent the upper and lower bounds of the $d$ th decision variable, respectively.

To define the Pareto optimal solution, four definitions are given as follows.

Pareto Dominance Relationship. A vector $X^{*}=\left[x_{1}^{*}, x_{2}^{*}, \ldots\right.$, $\left.x_{d}^{*}, \ldots, x_{D}^{*},\right]$ is said to dominate $X=\left[x_{1}, x_{2}, \ldots, x_{d}, \ldots, x_{D},\right]$ (known as $X^{*} \prec X$ ) if and only if the following two conditions are satisfied:

$$
\begin{array}{cl}
\forall n, & f_{n}\left(X^{*}\right) \leq f_{n}(X), \quad n=1,2, \ldots, \mathrm{N}, \\
\exists n_{0}, \quad f_{n_{0}}\left(X^{*}\right)<f_{n_{0}}(X), \quad 1 \leq n_{0} \leq \mathrm{N} .
\end{array}
$$

Pareto Optimal Solution. Pareto optimal solution is a solution that cannot be dominated by any solution in the feasible region $S$ which means that if and only if $\neg \exists X \in S: X \prec X^{*}$, $X^{*}$ is the Pareto optimal solution.

Pareto Optimal Set. For a given MOOP, the Pareto optimal set $P^{*}$ can be defined as

$$
P^{*}=\left\{X^{*} \in S \mid \neg \exists X \in S: X \prec X^{*}\right\}
$$

Pareto Optimal Front. For a given MOOP, the Pareto optimal front $P f^{*}$ can be described as

$$
\begin{aligned}
P f^{*} & =\left\{u=F(x)=\left(f_{1}(x), f_{2}(x), \ldots, f_{N}(x)\right)^{T} \mid X\right. \\
& \left.\in P^{*}\right\}
\end{aligned}
$$

\section{Problem Formulation of the MO-FOPID Problem under Multiworking Conditions}

To formulate the multiobjective FOPID controller optimization problem (MO-FOPID) under multiworking conditions, the fractional calculus theory and fractional-order PID (FOPID) controller are first introduced.

3.1. Introduction to Fractional Calculus Theory and FOPID Controller. Fractional calculus theory is an extension of the traditional calculus theory to the fractional systems. The fractional calculus theory can describe complex systems in an easy way with a clear physical meaning. Among the various fractional calculus operators, the Laplacian transformation defined by Caputo is the most usual mathematical expression for calculating the fractional-order time derivatives and has been widely used in fractional-order PID controller [28, 31]. Given a continuous derivable function $f(t)$, Caputo's fractional derivative of order $a$ can be defined as

$$
{ }_{0} D_{t}^{\alpha} f(t)=\frac{1}{\Gamma(n-\alpha)} \int_{0}^{t} \frac{f^{n}(t)}{(t-\tau)^{\alpha+1-n}} d \tau
$$

where ${ }_{0} D_{t}^{\alpha}$ denotes the fractional calculus operator and $\Gamma(\cdot)$ denotes the Euler Gamma function.

Fractional calculus equations usually need to be transformed into algebraic equations. The Laplacian transformation of (5) under zero initial condition can be expressed as

$$
\int_{0}^{\infty} e^{-s t} D^{\alpha} f(t) d t=s^{\alpha} F(s)
$$

In recent years, with the further research and development of fractional calculus theory, the combination of fractional calculus theory and modern control theory is becoming more and more popular. Controllers based on fractional calculus have been implemented and applied in many research fields $[12,25]$. The Oustaloup recursive filter and its improved version have been widely adopted to realize the discretization approximation of the fractional calculus operator. The expression of the Oustaloup filter is as follows:

$$
s^{\alpha} \approx K \prod_{k=-N}^{N}\left(\frac{\left(s+\omega_{k}^{\prime}\right)}{\left(s+\omega_{k}\right)}\right)
$$

where $\omega_{k}=\omega_{b}\left(\omega_{h} / \omega_{b}\right)^{(k+N+(1+\alpha) / 2) /(2 N+1)} ; \omega_{k}^{\prime}=\omega_{b}\left(\omega_{h} /\right.$ $\left.\omega_{b}\right)^{(k+N+(1-\alpha) / 2) /(2 N+1)} ; K=\omega_{h}^{\alpha} ; \alpha$ denotes the order of the fractional calculus; $(2 N+1)$ is the order of filter; $\left(\omega_{b}, \omega_{h}\right)$ denotes the expected fitting range; $N, \omega_{b}$, and $\omega_{h}$ are determined according to the accuracy requirement of the numerical approximation.

The FOPID controller proposed by Professor Podlubny [32] is an extension of the classical PID controller. Compared with the classical PID controller, the range of control rate of the FOPID controller is much wider. The transfer function of the FOPID controller is as follows:

$$
\frac{U(s)}{E(s)}=K_{p}+\frac{K_{i}}{s^{\lambda}}+K_{d} s^{u}
$$




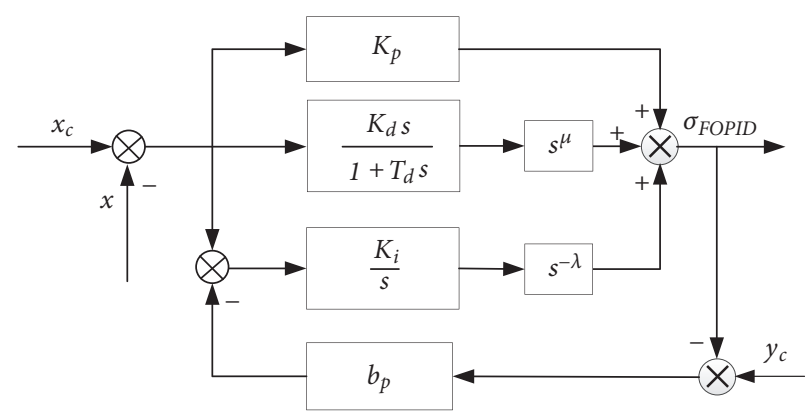

FIGURE 1: The structure of the FOPID controller for pumped turbine governing system.

where $E$ denotes the control deviation; $U$ denotes the controller output; $K_{p}, K_{i}$, and $K_{d}$ represent the gain parameter, the integral order, and the differential order, respectively. The traditional PID controller is a special case of the FOPID controller when $\lambda=1$ and $u=1$. Because the integral and differential orders are variational, the FOPID controller has better adaptability and flexibility and bigger potential to obtain better control performance [33]. The structure of the FOPID controller for PTGS is shown in Figure 1. In Figure $1, x_{c}$ denotes the given unit speed; $x$ denotes the unit speed; $b_{p}$ denotes the permanent slip coefficient; $T_{d}$ denotes the differential time constant; $K_{p}, K_{i}$, and $K_{d}$ represent the proportional, integral, and differential gain coefficients of the controller, respectively; $\lambda$ and $\mu$ denote the integral and differential orders of the controller, respectively.

3.2. Description of the PTGS System. PTGS is a complex nonlinear time-varying system of hydraulic, mechanical, and electrical connections. PTGS mainly contains five parts, namely, a controller, an electrohydraulic servomechanism system, a pump turbine, a generator, and a penstock system, where the controller and the servomechanism system consist of the speed governor of PTGS [4]. A PID controller has always been employed as part of the speed governor of PTGS. In this study, the FOPID controller is designed for PTGS. The FOPID controller has been described in Section 3.1. In what follows, transfer functions of the other four connectors are illuminated.

(1) Servomechanism System. Servomechanism is the actuator of the governor of PSU. It is made up of an auxiliary servomotor and a main servomotor of which the transfer functions are as follows:

$$
\begin{aligned}
G(s) & =\frac{K_{v}}{1+T_{y 1} s} \\
G_{v}(s) & =\frac{1}{T_{y} s}
\end{aligned}
$$

where $T_{y 1}$ and $K_{v}$ are the time constant and scale factor of the auxiliary servomotor, respectively, and $T_{y}$ represents the main servomotor open-loop time constant.
(2) Pump Turbine. To reflect the complex nonlinear characteristics among water, machine, and electricity during the operation process accurately, the pump turbine model based on characteristic curves is constructed. The model of pump turbine is as follows:

$$
\begin{aligned}
& M_{11}=f_{M}\left(a, N_{11}\right) \\
& Q_{11}=f_{\mathrm{Q}}\left(a, N_{11}\right)
\end{aligned}
$$

where $M_{11}, Q_{11}$, and $N_{11}$ represent the unit torque, unit flow, and unit speed, respectively; $a$ is the guide vane opening; $f_{M}$ and $f_{\mathrm{Q}}$ denote the functions of the moment and flow characteristic curves, respectively. Because of the strong nonlinear characteristics of the flow and moment characteristic curves, the improved Suter transform is introduced to transfer the flow and moment characteristic curves into $W H$ and $W M$ characteristic curves, respectively [4]. The $W H$ and $W M$ characteristic curves of a pump turbine in a pumped storage power station in China using the improved Suter transform have been illustrated in Zhou et al. [4]. In this study, the $W H$ and $W M$ characteristic curves in two dimensions in [4] have been changed to three dimensions. The three-dimensional $W H$ and $W M$ characteristic curves are illustrated as in Figure 2.

(3) Generator. The common first-order model $[6,16,18]$ is adopted in this study to balance the pump turbine torque and the generator torque. The transfer function of the first-order model is as follows:

$$
G_{g}(s)=\frac{1}{T_{a} s+e_{n}}
$$

where $T_{a}$ and $e_{n}$ are the inertia time constant and selfadjusting factor of the generator, respectively.

(4) Penstock System. Because of the fluid and tube wall elastic effects on the penstock, the second-order elastic water hammer model is exploited in this study by applying the second-order Taylor expansion on the nonlinear hyperbolic tangent function. The transfer function of the second-order elastic water hammer model is as follows:

$$
G_{h}(s)=\frac{H(s)}{Q(s)}=\frac{-T_{w} s}{1+0.5 f T_{r} s+0.125 T_{r}^{2} s^{2}}
$$




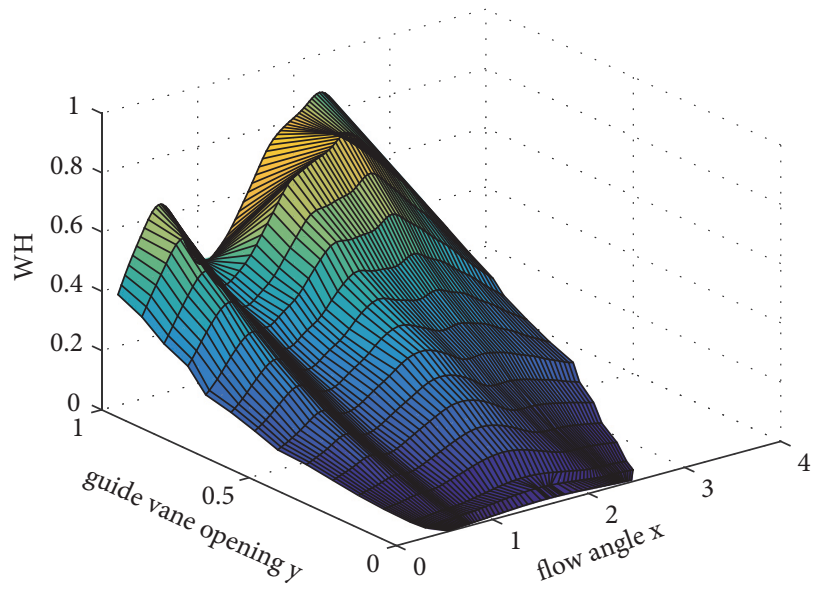

(a) WH characteristic curve

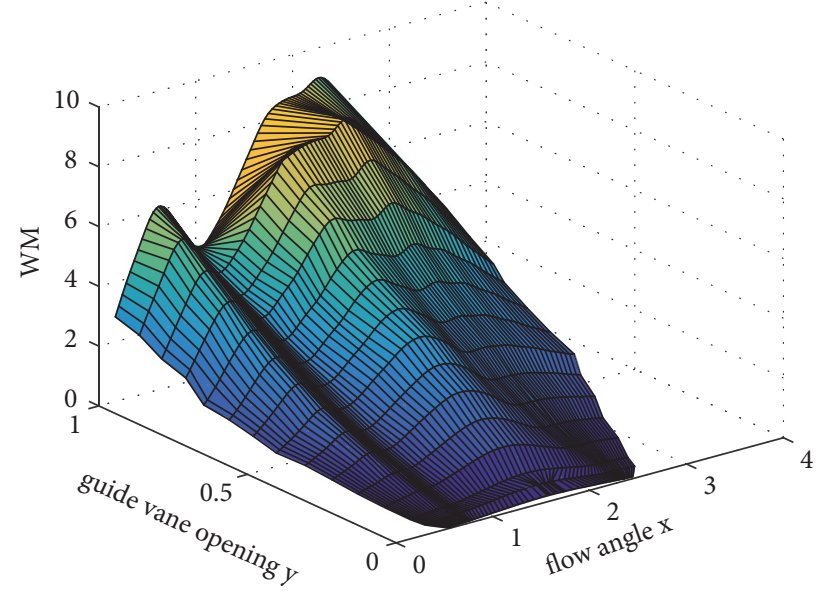

(b) WM characteristic curve

FIGURE 2: Three-dimensional surface of the characteristic curves of pump turbine.

where $T_{w}$ denotes water flow inertia time constant, $f$ represents the water head loss coefficient, and $T_{r}$ is the reflection time of water hammer wave.

\subsection{Multiobjective Optimization of FOPID Controller for} PTGS. For single-optimization of the FOPID controller under a certain working condition, the ITAE $[34,35]$ is usually employed as the objective function to obtain satisfactory transient dynamic performance of the system. The ITAE index is defined as follows:

$$
\operatorname{ITAE}=\int_{0}^{T} t|e(t)| d t
$$

where $e(t)$ denotes the relative deviation of the rotational speed of PTGS. The ITAE index considers the stable time and overshoot of the dynamic response of PTGS simultaneously. It can evaluate the speed and stability of the system at the same time. The smaller the ITAE, the better the speed and stability of the system.

When operating at the working condition of low water head, the PSU is easy to fall into the " $\mathrm{S}$ " characteristic region, resulting in oscillation of the unit speed near the rated frequency. The working condition of medium or high water head, in the other way, is the most common working condition in the operation process of PSU. The optimal control of PTGS under different working conditions should be considered and researched to make the PTGS system better adapt to the changeable working environment. However, the ITAE for PTGS at working condition of low head (referred as ITAE IT $_{1}$ ) and that of high head (referred as ITAE 2 ) usually influence and restrict each other. In this study, the single-objective optimization of the FOPID controller for PTGS is expanded to multiobjective theoretical framework to find a compromise solution and ensure that the PTGS system can achieve relatively better control performance under changeable working conditions. To formulate the MO-FOPID problem, the ITAE 1 and ITAE $_{2}$ are adopted as objective functions. The five parameters of the FOPID controller for PTGS including the proportional coefficient $K_{p}$, the integral coefficient $K_{i}$, the differential coefficient $K_{d}$, the integral order $\lambda$, and the differential order $\mu$ are taken as decision variables. The MO-FOPID problem can then be formulated as

$$
\begin{array}{ll}
\operatorname{Min} \quad & \left\{\begin{array}{l}
f_{1}=\operatorname{ITAE}_{1}=f_{1}\left(K_{p}, K_{i}, K_{d}, \lambda, \mu\right) \\
f_{2}=\operatorname{ITAE}_{2}=f_{2}\left(K_{p}, K_{i}, K_{d}, \lambda, \mu\right)
\end{array}\right. \\
\text { subject to }\left\{\begin{array}{l}
K_{p_{\text {min }}} \leq K_{p} \leq K_{p_{\text {max }}} \\
K_{i \text { min }} \leq K_{i} \leq K_{i_{\text {max }}} \\
K_{d_{\text {min }}} \leq K_{d} \leq K_{d \text { max }} \\
\lambda_{\text {min }} \leq \lambda \leq \lambda_{\max } \\
\mu_{\text {min }} \leq \mu \leq \mu_{\max }
\end{array}\right.
\end{array}
$$

where $f_{1}(\cdot)$ and $f_{2}(\cdot)$ are functions of $K_{p}, K_{i}, K_{d}, \lambda, \mu$ at working condition of low water head and high water head, respectively. $X_{\min }$ and $X_{\max }$ are the lower and upper bounds of $K_{p}, K_{i}, K_{d}, \lambda, \mu$ of the FOPID controller, respectively.

\section{Nondominated Sorting Genetic Algorithm-III Based on Latin-Hypercube Sampling and Chaos Theory (LCNSGA-III)}

An improved version of NSGA-III based on Latin-hypercube sampling and chaos theory is developed to solve the proposed MO-FOPID problem under multiworking conditions.

4.1. Brief Introduction to NSGA-III. The NSGA-III algorithm [36], first introduced by Deb and Jain in 2014, is a novel reference-point-based nondominated sorting genetic algorithm following the NSGA-II framework. Unlike the crowding distance operator exploited in NSGA-II [37], NSGAIII employed a reference point-based mechanism to make the Pareto optimal front well-distributed. The step-to-step 
procedures of the NSGA-III algorithm can be expressed as follows.

Step 1. Calculate the number of reference points $(H)$ to be placed on the hyper-plane.

$$
H=\left(\begin{array}{c}
C+g-1 \\
g
\end{array}\right)
$$

where $C$ represents the number of objective functions and $g$ denotes the number of divisions (For $C=3$ and $g=4, H$ is calculated as 15).

Step 2. Generate NP individuals in the feasible region randomly to form the initial population of the NSGA-III algorithm, and record it as $P_{k}$. Set the iteration number as $k=1$.

Step 3. Generate the offspring population $Q_{k}$ using the simulation binary crossover (SBX) operator and polynomial mutation operator.

Step 4. Let $R_{k}=Q_{k} \cup P_{k}$ and calculate the fitness value of each individual in $R_{k}$.

Step 5. Identify the nondominated level $F_{1}, F_{2}, \ldots, F_{t}$ for each individual in $R_{k}$ using the nondominated sorting operator.

Step 6. Normalize the objectives and associate the solutions in $R_{k}$ with the reference points. Delete the useless reference points and preserve solutions with higher rankings according to the niche preservation strategy to construct the next generation of population $P_{k+1}$.

Step 7. If $k<G_{\max }$, skip to Step 3; else stop iteration and output the Pareto optimal set.

The crossover and mutation operators in Step 3 and the nondominated sorting operator in Step 5 of the NSGA-III algorithm are the same as those of the NSGA-II algorithm. In Step 6, the NSGA-III algorithm generates the next population using the reference point-based selection mechanism other than the crowding distance operator of NSGA-II. Readers can refer to [36] for more details about NSGA-III. The improvements of the NSGA-III algorithms including the Latin hypercube sampling-based initialization technique and the chaotic crossover and mutation operators are introduced in the following subsections.

\subsection{Latin Hypercube Sampling Based Initialization Technique.} NSGA-III generates the initial values of the decision variables in the feasible region randomly to form the initial population of the algorithm. However, a large number of individuals may assemble into a local area of the feasible region because of the random initiation, leading to premature convergence in the iterative process. In order to make the individuals of the initial population well-distributed in the feasible region, the Latin hypercube sampling based initialization technique [38] is introduced to NSGA-III to improve its search performance.
The brief steps to generate the initial population of NSGA-III using Latin hypercube sampling are as follows.

Step 1. Suppose the size of the population is $N$ and each individual in the population contains $L$ discrete elements. The range of the discrete element $x_{l}, l=1,2, \ldots, L$ of individual $x$ can be divided into $N$ equal mini zones:

$$
x_{l\lrcorner \min }=x_{l}^{0}<x_{l}^{1}<\cdots<x_{l}^{j}<\cdots<x_{l}^{N}=x_{l\lrcorner \max }
$$

where $P\left(x_{l}^{j}<x<x_{l}^{j+1}\right)=1 / N$ and the value space of $x$ can be divided into $N^{L}$ small hypercubes finally.

Step 2. Generate a matrix $M$ of which the dimension is $N \times L$, and every column of $M$ is the full permutation of $\{1,2, \ldots, N\}$.

Step 3. Generate an individual in each row of $M$ randomly; an initial population with $N$ individuals is then generated.

4.3. Chaotic Crossover and Mutation Operators. The NSGAIII algorithm generates a random number $r_{c}$ to determine whether to apply the crossover operator or not. The SBX operator is implemented when $r_{c}<\eta_{c}$, where $\eta_{c}$ represents the crossover distribution index. For two parent individuals $x_{p 1}=\left\{x_{p 1}^{1}, \ldots, x_{p 1}^{i}, \ldots, x_{p 1}^{n}\right\}$ and $x_{p 2}=\left\{x_{p 2}^{1}, \ldots, x_{p 2}^{i}, \ldots\right.$, $\left.x_{p 2}^{n}\right\}$, the NSGA-III algorithm generates two offspring individuals $x_{c 1}=\left\{x_{c 1}^{1}, \ldots, x_{c 1}^{i}, \ldots, x_{c 1}^{n}\right\}$ and $x_{c 2}=\left\{x_{c 2}^{1}, \ldots, x_{c 2}^{i}\right.$, $\left.\ldots, x_{c 2}^{n}\right\}$ according to the following:

$$
\begin{aligned}
& x_{c 1}^{i}=\frac{1}{2}\left[(1-\beta) x_{p 1}^{i}+(1+\beta) x_{p 2}^{i}\right] \\
& x_{c 2}^{i}=\frac{1}{2}\left[(1+\beta) x_{p 1}^{i}+(1-\beta) x_{p 2}^{i}\right]
\end{aligned}
$$

where $\beta$ represents the crossover coefficient. $\beta$ can be calculated according to the following:

$$
\beta= \begin{cases}(2 u)^{1 /\left(\eta_{c}+1\right)}, & u \leq 0.5 \\ \left(\frac{1}{2(1-u)}\right)^{1 /\left(\eta_{c}+1\right)}, & \text { else }\end{cases}
$$

where $u=\operatorname{rand}(\cdot)$ is a random number uniformly generated in $[0,1]$.

The NSGA-III algorithm generates a random number $r_{m}$ to determine whether to apply the mutation operator or not. The polynomial mutation operator is implemented when $r_{m}<\eta_{m}$, where $\eta_{m}$ represents the mutation distribution index. For the feasible solution $x_{s}$, the mutation individual is generated using the polynomial mutation operator:

$$
x_{s}^{*}=x_{s}+\left(x_{s}^{u}-x_{s}^{l}\right) \times \delta_{s}
$$

where $x_{s}^{*}$ represents the mutation individual; $x_{s}^{u}$ and $x_{s}^{l}$ represent the upper and lower bounds of $x_{s}$, respectively; $\delta_{s}$ is the mutation coefficient; $\delta_{s}$ can be calculated as follows:

$$
\delta_{s}= \begin{cases}\left(2 u_{s}\right)^{1 /\left(\eta_{m}+1\right)}-1, & u_{s}<0.5 \\ 1-\left(2 \times\left(1-u_{s}\right)\right)^{1 /\left(\eta_{m}+1\right)}, & \text { else }\end{cases}
$$


where $u_{s}=\operatorname{rand}(\cdot)$ is a random number uniformly generated in $[0,1]$.

The standard NSGA-III algorithm has excellent computational efficiency and stability. However, the NSGA-III algorithm sometimes may fall into the local optimal solution because of the insufficient exploration of the feasible region. Because of the ergodicity and stochasticity of chaotic sequences [39], the chaotic map which can generate chaotic sequences is introduced into the crossover and mutation operators of NSGA-III to enhance its global search and local exploration ability [40]. In this study, the tent map [41] is employed to improve the crossover and mutation operators of NSGA-III.

The tent chaotic map with uniform distribution probability density can be expressed as

$$
c x^{(k+1)}= \begin{cases}\frac{c x^{(k)}}{0.5} & c x^{(k)}<0.5 \\ 2 \cdot\left(1-c x^{(k)}\right) & \text { else }\end{cases}
$$

where $x^{(k)} \in(0,1)$ denotes the chaotic variable generated in the $k$ th iteration.

According to (19) and (21), the crossover and mutation operators of the standard NSGA-III algorithm need to generate two random numbers $u$ and $u_{s}$ in $[0,1]$, respectively. The two numbers $u$ and $u_{s}$ are generated using the tent chaotic map in the LCNSGA-III algorithm. The two random numbers $u$ and $u_{s}$ are generated according to the following:

$$
\begin{gathered}
u=c x^{(k+1)} \\
u_{s}=c x^{(k+1)}
\end{gathered}
$$

4.4. The Flowchart of LCNSGA-III for MOOPs. Based on the above introduction of LCNSGA-III, the Latin hypercube sampling, and the chaotic crossover and mutation operators, the flowchart of LCNSGA-III for MOOPs is shown in Figure 3.

4.5. Implementation of LCNSGA-III for Solving the MOFOPID Problem under Multiworking Conditions. The above LCNSGA-III algorithm is used to optimize the FOPID controller for PTGS under multiworking conditions. The schematic diagram of the MO-FOPID problem optimized by LCNSGA-III under multiworking conditions is shown in Figure 4. In Figure 4, $x_{c}$ denotes the rotational speed (or frequency) and $y_{c}$ denotes the given guide vane opening. Since the frequency of the rotational speed is $50 \mathrm{~Hz}$ and the frequency disturbance is $2 \mathrm{~Hz}$, the frequency perturbation is set as $4 \%$ of the rated frequency.

\section{Numerical Experiments and Analysis}

\subsection{Experiments for Benchmark Functions}

5.1.1. Benchmark Functions and Performance Metrics. In order to validity the effectiveness of the newly developed LCNSGA-III algorithm based on Latin-hypercube sampling

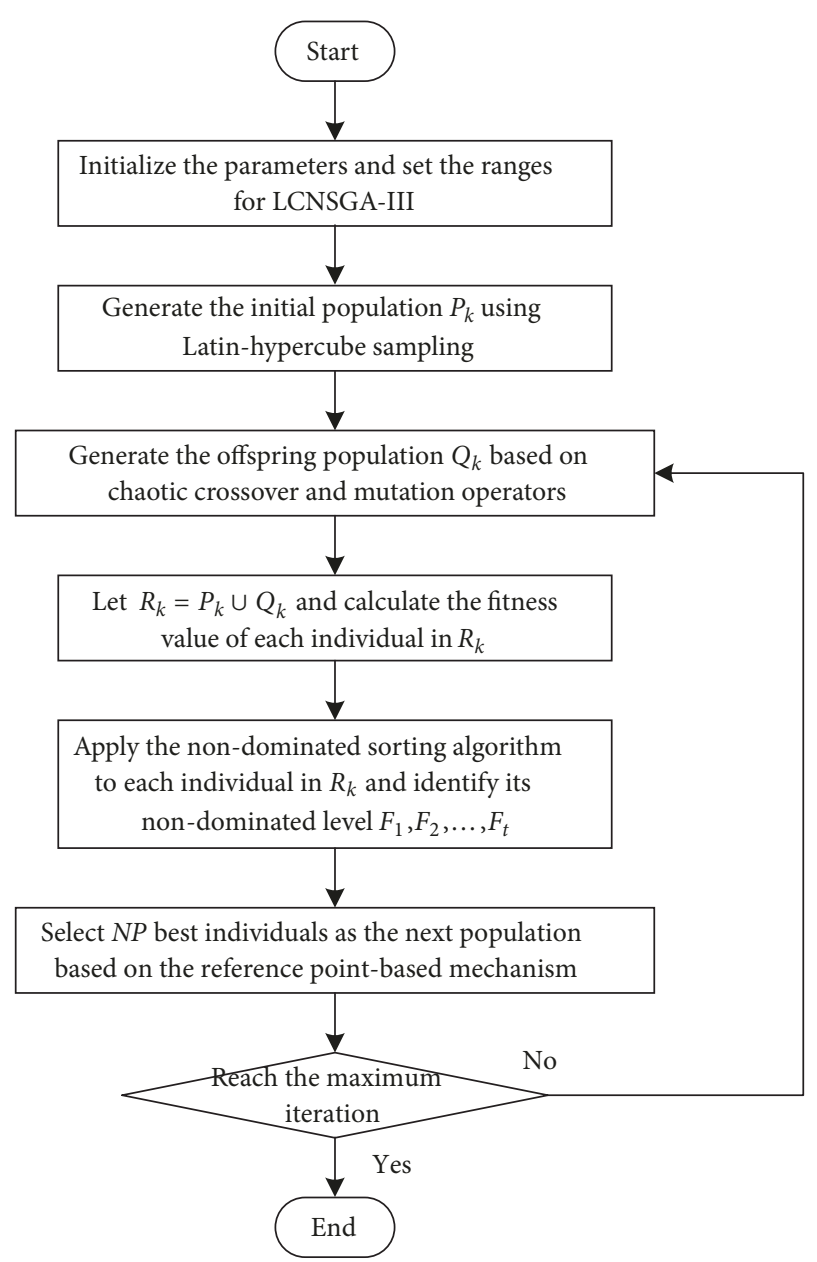

FIgURE 3: Flowchart of LCNSGA-III for MOOPs.

and chaotic map, a total number of eight test functions including the ZDT1-4, 6 [42] and the DTLZ1-2, 5 [43] (shown in Table 1) are employed to test its performance. Among the eight test functions for MOOPs, the ZDT benchmark functions are two-objective MOOPs. The dimension of the decision variables and the number of the test functions of the DTLZ benchmark functions can be adjusted. In this study, the number of the objective functions of the DTLZ test functions is selected as three to display the Pareto front of threeobjective MOOPs. Four other typical multiobjective algorithms including NSGA-II, NSGA-III, MOEA/D, and PESAII $[30,44]$ are adopted as control group. The performances of the multiobjective evolutionary algorithms (MOEAs) are evaluated and compared using the widely used evaluation metrics of generational distance (GD) and Spread. GD is adopted to measure the mean value of the distance between the Pareto solution set and the real Pareto front. Spread is employed to describe the distribution uniformity of the Pareto optimal set [40]. GD can be expressed as follows:

$$
\mathrm{GD}=\frac{1}{N} \sqrt{\sum_{i=1}^{N} D_{i}^{2}}
$$




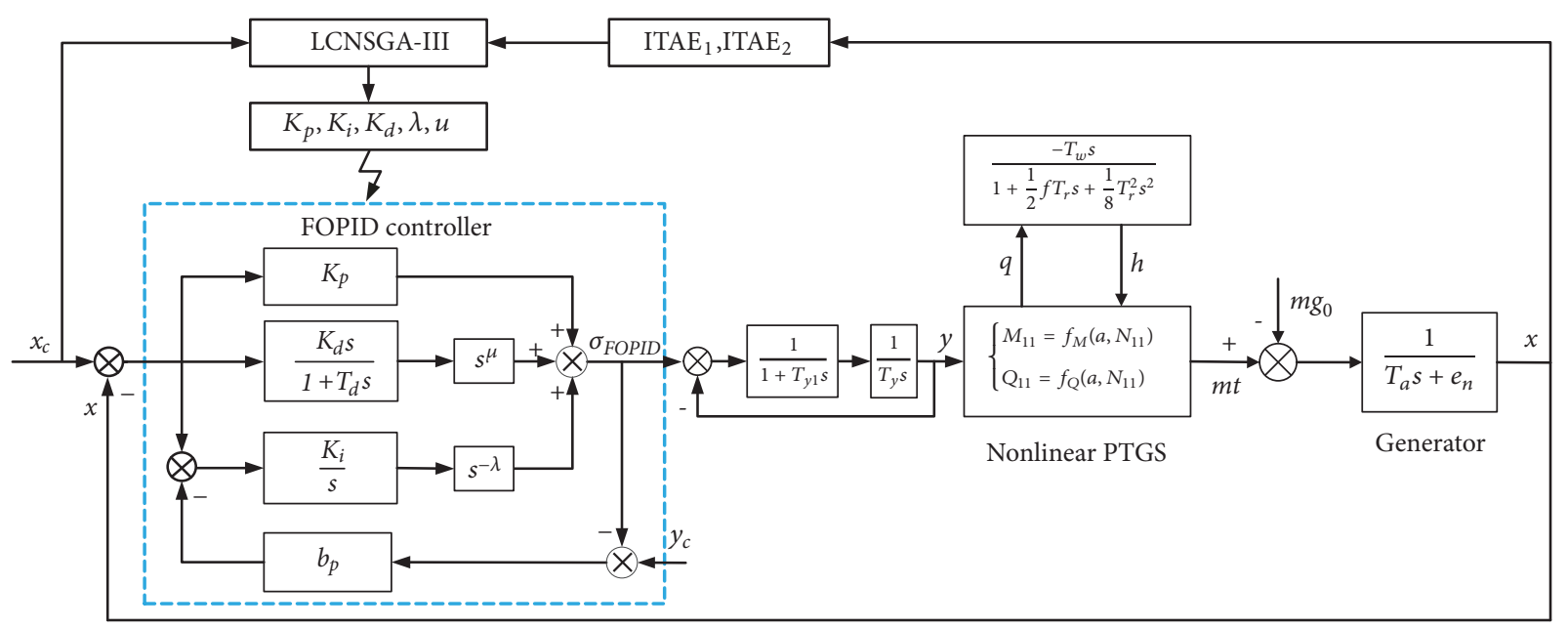

FIGURE 4: Schematic diagram of the MO-FOPID problem optimized by LCNSGA-III under multiworking conditions.

where $D_{i}$ denotes the Euclidean distance between the $i$ th nondominated solution and the nearest nondominated solution on the real Pareto front and $N$ denotes the size of the Pareto optimal set. The smaller the GD, the closer the Pareto solution set to the real Pareto front and the better the convergence accuracy.

Spread can be expressed as follows:

$$
\text { Spread }=\frac{\sum_{j=1}^{M} d_{j}^{e}+\sum_{i=1}^{N}\left|d_{i}-\bar{d}\right|}{\sum_{j=1}^{M} d_{j}^{e}+N \cdot \bar{d}}
$$

where $d_{i}$ denotes the distance between $i$ th solution with its neighboring solution; $\bar{d}$ is the mean of $d_{i}$; $d_{j}^{e}$ denotes the Euclidean distance between the extreme solution of the real Pareto front and the boundary solution of the obtained Pareto optimal set; $M$ denotes the number of objective functions. The smaller the Spread, the better the distribution of the solutions.

5.1.2. Results Analysis. The parameters of the five algorithms for MOOPs with two objectives are set as follows: the population size is set as 100 while the number of iterations is set as 300 . The parameters of the five algorithms for MOOPs with three objectives are set as follows: the population size is set as 150 while the number of iterations is 500 . The crossover and mutation probability of the five algorithms are set as 0.7 and 0.3 , respectively. The neighborhood size of MOEA/D is set as $20[45,46]$. All the experiments are implemented in Matlab environment. Because of the random initialization of the MOOPs, all the experiments have been repeated ten times independently to eliminate the effectiveness of randomness. The average GD and Spread of the five algorithms for the eight benchmark functions are given in Tables 2 and 3, respectively. Numbers in bold represent the optimal GD and Spread among the five algorithms for the eight benchmark functions.

As can be seen from Table 2, the GD of the Pareto optimal solution obtained by the newly developed LCNSGA-III is the best out of the total eight benchmark functions. Although the GD of LCNSGA-III algorithm is slightly worse than that of NSGA-II and NSGA-III to optimize ZDT4 and DTLZ1, the Spread of LCNSGA-III is prominent. The Spread of LCNSGA-III performs best to optimize the ZDT1, ZDT4, and DTLZ5 benchmark problems. And the Spread for LCNSGAIII does not differ much from those for the other five MOOPs except DTLZ1, which indicates that the LCNSGA-III algorithm can obtain good convergence performance for the eight benchmark functions. The Pareto optimal front obtained by LCNSGA-III to optimize the eight benchmark functions is shown in Figure 5 (ZDT1-4) and Figure 6 (ZDT6, DTLZ1-2, 5) (Grey points denote the true Pareto front while blue ones denote the obtained Pareto front), respectively. As depicted in Figures 5 and 6, the Pareto front obtained by LCNSGA-III to optimize the eight benchmark MOOPs can approximate the real Pareto front perfectly and the distributions of the Pareto optimal sets are uniform. As a result, the LCNSGAIII algorithm has prominent convergence performance and optimization ability compared with the other algorithms.

\subsection{Experiments for Nonlinear PTGS}

5.2.1. Experiments Design and Results. In order to fully verify the performance and effectiveness of the LCNSGAIII algorithm in solving the MO-FOPID problem under multiworking conditions, the nonlinear model of PTGS with different controllers (PID and FOPID) and water heads $(198 \mathrm{~m}, 205 \mathrm{~m}$, and $210 \mathrm{~m})$ under no-load conditions is simulated on MATLAB environment. The frequency perturbation is set as $4 \%$ of the rated frequency and the simulation time is set as 50s. A total number of ten schemes have been designed to obtain the optimal parameters. The ten schemes can be divided into four categories as follows:

(1) The backtracking search algorithm (BSA) [47] is exploited to optimize the parameters of PID controller under a single working condition of different water heads $(198 \mathrm{~m}$, $205 \mathrm{~m}$, and $210 \mathrm{~m})$. The ITAE of PTGS under a certain working condition is selected as the objective function for 
TABLE 1: Eight test functions for MOOPs.

\begin{tabular}{|c|c|c|c|c|}
\hline Name & Functions & $\begin{array}{c}\text { Dimension of } \\
\text { decision variable }\end{array}$ & Feasible region & Type of Pareto Front \\
\hline ZDT1 & $\begin{array}{c}f_{1}(X)=x_{1}, \\
f_{2}(X)=g \cdot\left(1-\sqrt{\frac{f_{1}}{g}}\right) \\
g(X)=1+9 \cdot \sum_{i=2}^{n} \frac{x_{i}}{(n-1)}\end{array}$ & 30 & {$[0,1]$} & High dimension, convex \\
\hline ZDT2 & $\begin{array}{c}f_{1}(X)=x_{1} \\
f_{2}(X)=g \cdot\left(1-\left(\frac{f_{2}}{g}\right)^{2}\right) \\
g(X)=1+9 \cdot \sum_{i=2}^{n} \frac{x_{i}}{(n-1)}\end{array}$ & 30 & {$[0,1]$} & High dimension, convex \\
\hline ZDT3 & $\begin{array}{c}f_{1}(X)=x_{1} \\
\left.f_{2}(X)=g \cdot\left(1-\sqrt{\frac{f_{1}}{g}}\right)-\left(\frac{f_{1}}{g}\right) \sin \left(10 \pi f_{1}\right)\right) \\
g(X)=1+9 \cdot \sum_{i=2}^{n} \frac{x_{i}}{(n-1)}\end{array}$ & 30 & {$[0,1]$} & Discontinuous, convex \\
\hline ZDT4 & $\begin{array}{c}f_{1}(X)=x_{1} \\
f_{2}(X)=g \cdot\left(1-\sqrt{\frac{f_{1}}{g}}\right) \\
g(X)=1+10(n-1)+\sum_{i=2}^{n}\left[x_{i}^{2}-10 \cos \left(4 \pi x_{i}\right)\right]\end{array}$ & 10 & $\begin{array}{l}x_{1} \in[0,1] \\
x_{i} \in[-5,5] \\
i=2,3, \ldots, n\end{array}$ & Multi-modal, convex \\
\hline ZDT6 & $\begin{array}{c}f_{1}(X)=1-\exp \left(-4 x_{1}\right) \sin ^{6}\left(6 \pi x_{1}\right) \\
f_{2}(X)=g \cdot\left(1-\left(\frac{f_{1}}{g}\right)^{2}\right) \\
g(X)=1+9 \cdot\left[\sum_{i=2}^{n} \frac{x_{i}}{(n-1)}\right]^{0.25}\end{array}$ & 10 & {$[0,1]$} & Inhomogeneous \\
\hline DTLZ1 & $\begin{array}{c}f_{1}(X)=\frac{1}{2} x_{1} x_{2} \cdots x_{M-1}\left(1+g\left(x_{M}\right)\right) \\
f_{2}(X)=\frac{1}{2} x_{1} x_{2} \cdots\left(1-x_{M-1}\right)\left(1+g\left(x_{M}\right)\right) \\
\vdots \\
f_{M-1}(X)=\frac{1}{2} x_{1}\left(1-x_{2}\right)\left(1+g\left(x_{M}\right)\right) \\
f_{M}(X)=\frac{1}{2}\left(1-x_{1}\right)\left(1+g\left(x_{M}\right)\right) \\
g\left(X_{M}\right)= \\
100\left(x_{M}+\sum_{x_{i} \in x_{M}} *(x-0.5)^{2}+\cos (20 \pi(x-0.5))\right)\end{array}$ & 7 & {$[0,1]$} & Linear, multimodal \\
\hline DTLZ2 & $\begin{array}{c}f_{1}(X)=\left(1+g\left(x_{M}\right)\right) \cos \left(\frac{x_{2} \pi}{2}\right) \cdots \cos \left(\frac{x_{M-1} \pi}{2}\right) \\
f_{2}(X)=\left(1+g\left(x_{M}\right)\right) \cos \left(\frac{x_{1} \pi}{2}\right) \cdots \cos \left(\frac{x_{M-1} \pi}{2}\right) \\
\vdots \\
f_{M}(X)=\left(1+g\left(x_{M}\right)\right) \sin \left(\frac{x_{1} \pi}{2}\right) \\
\left.g\left(X_{M}\right)=\sum *(x-0.5)^{2}\right)\end{array}$ & 12 & {$[0,1]$} & Complex nonconvex \\
\hline DTLZ5 & $\begin{array}{c}\text { replace the } x_{i} \text { in DTLZ2 with } \theta_{i} \\
\theta_{i}=\frac{\pi}{4(1+q(r))}\left(1+2 g(r) x_{i}\right) \\
g\left(x_{M}\right)=\Sigma_{x_{i} \in x_{M}} x_{i}^{0.1}\end{array}$ & 12 & {$[0,1]$} & Space arc \\
\hline
\end{tabular}


TABLE 2: Comparison of GD for eight benchmark functions.

\begin{tabular}{|c|c|c|c|c|c|}
\hline \multirow{2}{*}{ Benchmark functions } & \multicolumn{5}{|c|}{ Multi-objective optimization algorithms } \\
\hline & PESA-II & MOEA/D & NSGA-II & NSGA-III & LCNSGA-III \\
\hline ZDT1 & $7.72 \mathrm{E}-05$ & $3.66 \mathrm{E}-04$ & $5.77 \mathrm{E}-05$ & 7.95E-05 & $3.80 E-05$ \\
\hline ZDT2 & 3.37E-04 & 8.27E-04 & 3.11E-05 & $6.77 \mathrm{E}-05$ & $2.84 E-05$ \\
\hline ZDT3 & $6.14 \mathrm{E}-05$ & $1.90 \mathrm{E}-03$ & 4.23E-05 & $7.48 \mathrm{E}-05$ & $3.56 E-05$ \\
\hline ZDT4 & 1.41E-02 & $2.05 \mathrm{E}-03$ & $2.00 E-04$ & $2.52 \mathrm{E}-04$ & $3.15 \mathrm{E}-04$ \\
\hline ZDT6 & $1.09 \mathrm{E}-02$ & $7.38 \mathrm{E}-04$ & 4.30E-05 & $7.84 \mathrm{E}-05$ & $3.63 E-05$ \\
\hline DTLZ1 & $1.97 \mathrm{E}-02$ & $3.01 \mathrm{E}-04$ & $4.75 \mathrm{E}-04$ & $2.25 E-04$ & 4.33E-04 \\
\hline DTLZ2 & 9.77E-04 & 4.18E-04 & $9.30 \mathrm{E}-04$ & 4.14E-04 & $3.78 E-04$ \\
\hline DTLZ5 & $1.50 \mathrm{E}-04$ & 7.88E-05 & $1.32 \mathrm{E}-04$ & $1.55 \mathrm{E}-04$ & $5.34 E-05$ \\
\hline
\end{tabular}

TABle 3: Comparison of Spread for eight benchmark functions.

\begin{tabular}{|c|c|c|c|c|c|}
\hline \multirow{2}{*}{ Benchmark functions } & \multicolumn{5}{|c|}{ Multi-objective optimization algorithms } \\
\hline & PESA-II & $\mathrm{MOEA} / \mathrm{D}$ & NSGA-II & NSGA-III & LCNSGA-III \\
\hline ZDT1 & $9.67 \mathrm{E}-01$ & $4.53 \mathrm{E}-01$ & $4.25 \mathrm{E}-01$ & $3.47 \mathrm{E}-01$ & $3.24 E-01$ \\
\hline ZDT2 & $9.83 \mathrm{E}-01$ & $6.73 \mathrm{E}-01$ & 4.68E-01 & $2.15 E-01$ & 2.99E-01 \\
\hline ZDT3 & $1.01 \mathrm{E}+00$ & $6.79 \mathrm{E}-01$ & $5.87 E-01$ & $7.52 \mathrm{E}-01$ & $6.65 \mathrm{E}-01$ \\
\hline ZDT4 & $1.02 \mathrm{E}+00$ & $5.51 \mathrm{E}-01$ & 4.31E-01 & $3.93 \mathrm{E}-01$ & $3.30 E-01$ \\
\hline ZDT6 & $1.04 \mathrm{E}+00$ & $1.55 \mathrm{E}-01$ & $4.15 \mathrm{E}-01$ & $1.13 E-01$ & $1.20 \mathrm{E}-01$ \\
\hline DTLZ1 & $8.02 \mathrm{E}-01$ & $3.11 E-02$ & 4.95E-01 & $3.40 \mathrm{E}-02$ & $3.51 \mathrm{E}-01$ \\
\hline DTLZ2 & $3.88 \mathrm{E}-01$ & $1.73 E-01$ & $5.21 \mathrm{E}-01$ & $1.74 \mathrm{E}-01$ & 3.01E-01 \\
\hline DTLZ5 & $9.20 \mathrm{E}-01$ & $2.03 \mathrm{E}+00$ & $6.61 \mathrm{E}-01$ & $9.78 \mathrm{E}-01$ & $4.58 E-01$ \\
\hline
\end{tabular}
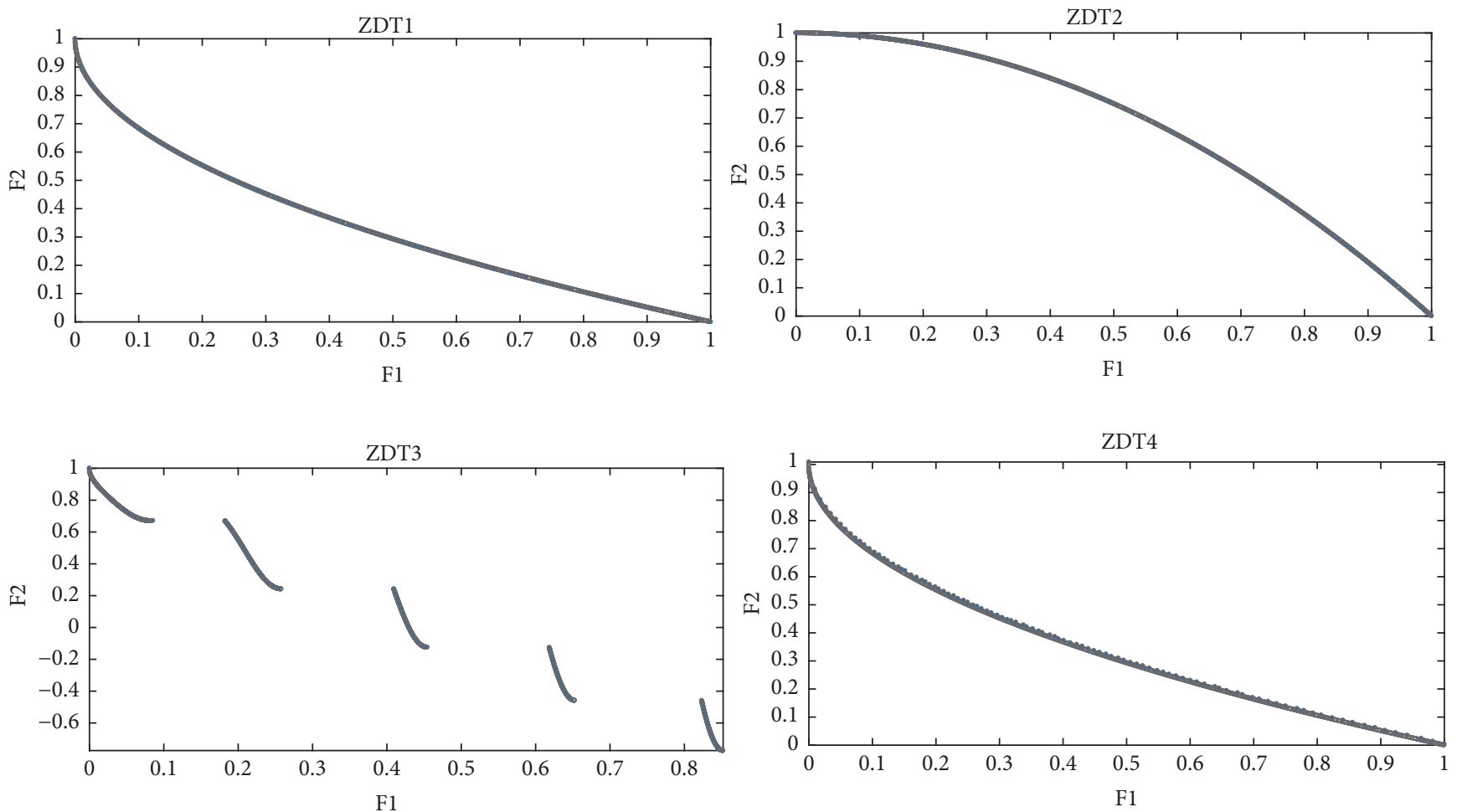

FIGURE 5: Pareto optimal solutions obtained by LCNSGA-III for ZDT1-4. 

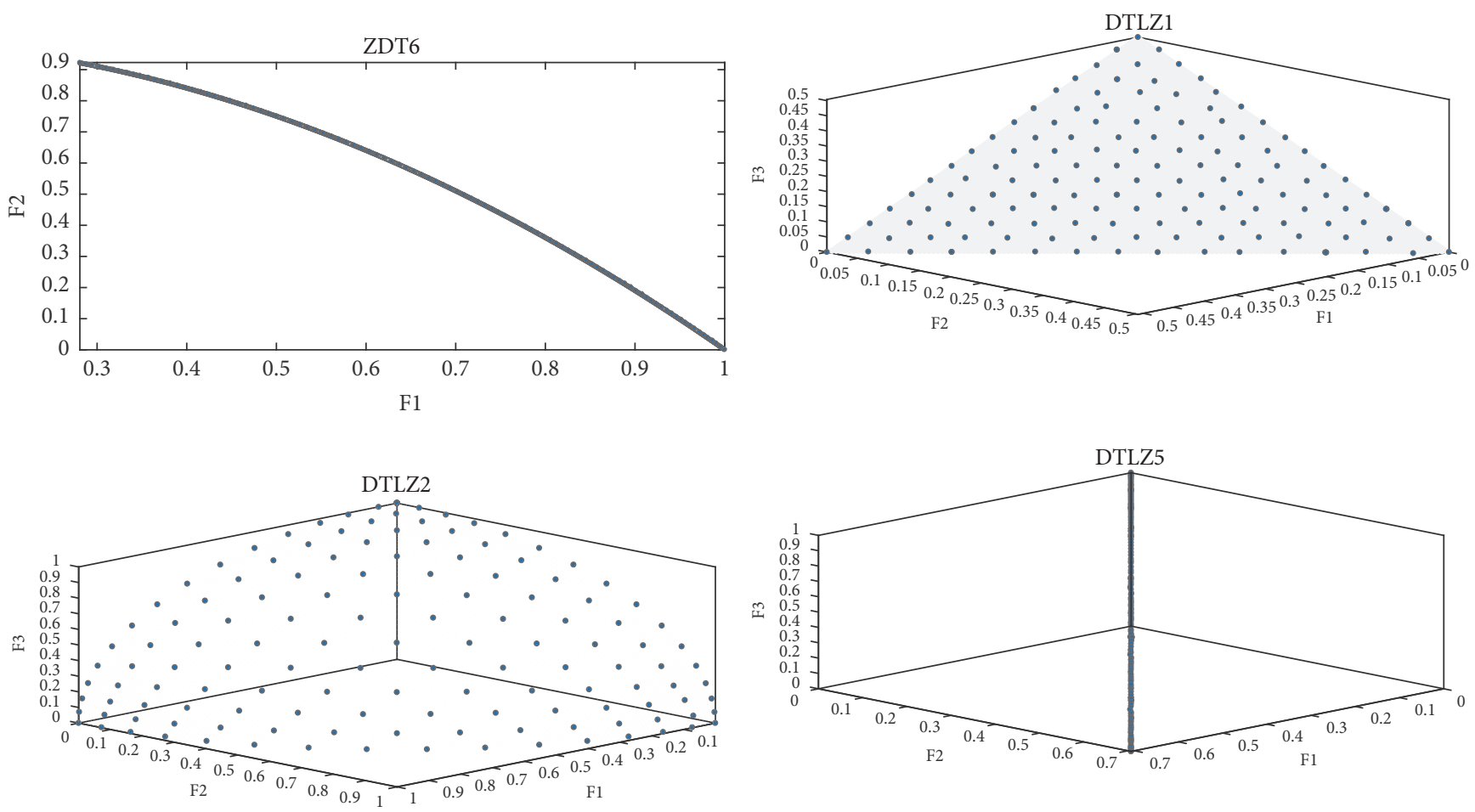

Figure 6: Pareto optimal solutions obtained by LCNSGA-III for ZDT6, DTLZ1-2, 5.

single-objective optimization. The experiments are simplified as S-198-PID, S-205-PID, and S-210-PID, respectively. The optimized control parameters are applied on the other two working conditions to test the adaptability of different schemes in tracking the dynamic responses of PTGS. (e.g., for scheme S-198-PID, the working condition running at $198 \mathrm{~m}$ water head is used for training while those at $205 \mathrm{~m}$ and $210 \mathrm{~m}$ are used for testing);

(2) The BSA algorithm is exploited to optimize the parameters of FOPID controller under a single working condition of different water heads $(198 \mathrm{~m}, 205 \mathrm{~m}$, and $210 \mathrm{~m})$. The experiments are simplified as S-198-FOPID, S-205-FOPID, and S210-FOPID, respectively. The optimized control parameters are applied on the other two working conditions to test the adaptability of different schemes in tracking the dynamic responses of PTGS;

(3) The NSGA-III algorithm is adopted to optimize the parameters of PID or FOPID controller under two working conditions (198m and 210m). The ITAE of PTGS at working conditions of $198 \mathrm{~m}$ and $210 \mathrm{~m}$ water head is selected as the objective functions for multiobjective optimization, and the compromise optimal solution among the Pareto optimal set is selected. The experiments are simplified as NSGA-III-PID and NSGA-III-FOPID for the PID and FOPID controller, respectively. The compromise optimal control parameters are applied on the working condition of $205 \mathrm{~m}$ water head to test the adaptability of different schemes in tracking the dynamic responses of PTGS;

(4) The LCNSGA-III algorithm is adopted to optimize the parameters of PID or FOPID controller under two working conditions (198m and $210 \mathrm{~m})$. The experiments are simplified as LCNSGA-III-PID and LCNSGA-III-FOPID for the PID and FOPID controller, respectively. The compromise optimal control parameters are applied on the working condition of $205 \mathrm{~m}$ water head to test the adaptability of different schemes in tracking the dynamic responses of PTGS.

The parameters of PTGS are set as follows: the ranges of $K_{p}, K_{i}$, and $K_{d}$ of the PID and FOPID controllers are all set as $[0,15]$ and the ranges of $\lambda$ and $u$ are all set as $[0,2]$. The parameters of the BSA algorithm are set as follows: the population size is set as 50 , the number of iterations is 200 , and the control parameter $F$ is set as the default value. The parameters of the NSGA-III and LCNSGA-III algorithms are as follows: the population size is set as 50 ; the iteration number is 300 ; the crossover probability is 0.7 ; the mutation probability is 0.3 .

Apart from ITAE, the integral of the ITSE [29], the stable time (ST) and the overshoot (OSO) is adopted to evaluate the performance of different schemes. The ITSE index is defined as follows:

$$
\operatorname{ITSE}=\int_{0}^{T} t(e(t))^{2} d t
$$

In this study, the best compromise solution is selected according to the subjective weighting method based on experts' preferences of weights. The optimal control parameters of the ten schemes for PTGS are shown in Table 4. The performance indices including ITAE, ITSE, ST, and OSO of the ten schemes under different working water heads are shown in Table 5. In Table 5, for scheme S-198-PID, the results for $198 \mathrm{~m}$ water head are training results while those for $205 \mathrm{~m}$ and $210 \mathrm{~m}$ heads are testing results. For scheme S-205-PID, the 
TABLE 4: Optimal control parameters for nonlinear PTGS using different schemes.

\begin{tabular}{|c|c|c|c|c|c|}
\hline \multirow{2}{*}{ Schemes } & \multicolumn{5}{|c|}{ Optimal parameters } \\
\hline & $K_{p}$ & $K_{i}$ & $K_{d}$ & $\lambda$ & $\mu$ \\
\hline S-198-PID & 5.05 & 0.72 & 2.08 & 1 & 1 \\
\hline S-205-PID & 8.00 & 1.00 & 2.91 & l & l \\
\hline S-210-PID & 6.97 & 0.87 & 5.00 & l & l \\
\hline S-198-FOPID & 9.00 & 0.52 & 0.82 & 0.49 & 0.97 \\
\hline S-205-FOPID & 1.36 & 0.58 & 1.74 & 0.64 & 0.98 \\
\hline S-210-FOPID & 8.91 & 0.56 & 1.98 & 0.77 & 0.99 \\
\hline NSGA-III-PID & 9.92 & 1.04 & 1.62 & I & l \\
\hline NSGA-III-FOPID & 8.90 & 0.52 & 1.02 & 0.57 & 0.98 \\
\hline LCNSGA-III-PID & 14.72 & 0.97 & 0.81 & l & l \\
\hline LCNSGA-III-FOPID & 10.62 & 0.58 & 1.12 & 0.56 & 0.98 \\
\hline
\end{tabular}

TABLE 5: Performance indices for nonlinear PTGS using different schemes at different water heads.

\begin{tabular}{lcccccccccccc}
\hline \multirow{2}{*}{ Experiments } & \multicolumn{4}{c}{$198 \mathrm{~m}$} & \multicolumn{4}{c}{ 205m } & \multicolumn{3}{c}{$210 \mathrm{~m}$} \\
& ITAE & ITSE & ST (s) & OSO (\%) & ITAE & ITSE & ST (s) & OSO (\%) & ITAE & ITSE & ST (s) & OSO (\%) \\
\hline S-198-PID & 5.68 & 0.04 & 49.9 & 17.7 & 4.10 & 0.02 & 35.6 & 15.0 & 3.16 & 0.02 & 35.5 & 12.4 \\
S-205-PID & 37.28 & 0.17 & $/$ & 25.4 & 2.69 & 0.02 & 29.3 & 22.9 & 1.93 & 0.02 & 20.1 & 20.5 \\
S-210-PID & 54.29 & 0.35 & $/$ & 22.8 & 37.88 & 0.21 & $/$ & 11.7 & 1.34 & 0.02 & 18.6 & 9.3 \\
S-198-FOPID & 2.10 & 0.02 & 27.6 & 3.6 & 1.50 & 0.02 & 26.1 & 0.5 & 1.87 & 0.02 & 27.1 & 0.9 \\
S-205-FOPID & 23.29 & 0.10 & $/$ & 10.5 & 1.11 & 0.02 & 25.0 & 0.5 & 1.48 & 0.01 & 28.2 & 0.7 \\
S-210-FOPID & 20.11 & 0.08 & $/$ & 7.9 & 1.81 & 0.02 & 25.7 & 3.3 & 0.96 & 0.02 & 17.0 & 0.3 \\
NSGA-III-PID & 5.88 & 0.04 & $/$ & 37.1 & 2.82 & 0.03 & 23.2 & 34.5 & 2.04 & 0.02 & 23.7 & 32.4 \\
NSGA-III-FOPID & 2.18 & 0.02 & 27.4 & 5.6 & 1.52 & 0.02 & 24.1 & 3.0 & 1.41 & 0.02 & 26.6 & 0.9 \\
LCNSGA-III-PID & 5.43 & 0.04 & $/$ & 33.3 & 2.76 & 0.03 & 18.4 & 30.4 & 1.90 & 0.02 & 18.4 & 27.6 \\
LCNSGA-III-FOPID & 1.90 & 0.02 & 27.6 & 5.5 & 1.37 & 0.02 & 23.3 & 2.3 & 1.42 & 0.01 & 25.9 & 1.1 \\
\hline
\end{tabular}

results for $205 \mathrm{~m}$ water head are training results while those for $198 \mathrm{~m}$ and $210 \mathrm{~m}$ heads are testing results. For scheme S210 -PID, the results for $210 \mathrm{~m}$ water head are training results while those for $198 \mathrm{~m}$ and $205 \mathrm{~m}$ heads are testing results. The single-objective schemes are designed to compare with the multiobjective schemes to highlight the effectiveness of multiobjective schemes in optimizing PTGS. "/" means that the system is unstable and when the fluctuation of frequency is smaller than 0.003 , it is considered to be stable.

5.2.2. Comparison of PID and FOPID Controllers under Different Working Conditions. From Table 5, it is known that the FOPID controller generally achieves better performance than the corresponding PID controller in terms of ITAE, ITSE ST, and OSO for $198 \mathrm{~m}, 205 \mathrm{~m}$, and $210 \mathrm{~m}$ working water heads. The ITAE, ITSE ST, and OSO for the FOPID controller are either smaller or in coincidence with those of the PID controller. For example, the improved percentages of scheme S-198-FOPID compared with scheme S-198-PID are $63.0 \%$, $50.0 \%, 44.7 \%$, and $79.7 \%$ in terms of ITAE, ITSE, ST, and OSO, respectively in the training stage $(198 \mathrm{~m})$. The improved percentages in the testing stage $(205 \mathrm{~m})$ are $63.4 \%, 26.7 \%$, and $96.7 \%$ in terms of ITAE, ST, and OSO, respectively. The ITSE for S-198-FOPID and scheme S-198-PID in the testing stage $(205 \mathrm{~m})$ is the same. To further compare the effects of different controllers in capturing the dynamic performances of PTGS, the test unit frequency at $198 \mathrm{~m}, 205 \mathrm{~m}$, and $210 \mathrm{~m}$ working water heads using different controllers is illustrated in Figures $7-9$, respectively. As can be seen from Figures 7-9, the FOPID controller for PTGS can obtain smaller overshoot ITAE, ITSE, ST, and OSO in most cases. For working condition of $198 \mathrm{~m}$ water head which is easy to fall into the " $\mathrm{S}$ " area, the unit frequency oscillates a lot using PID controller. The FOPID controller, in the other way, can effectively restrain the strong nonlinear characteristics of PTGS and significantly improve the control quality.

5.2.3. Analysis of Controllers Optimized under a Single Working Condition. It is noticed from Table 5 that for controllers optimized at a single working condition, the performance is only good for the training working condition, but the test working conditions. It can also be seen from Table 5 and Figures 7-9 that the S-210-FOPID scheme can obtain good control performance when PTGS is running at $210 \mathrm{~m}$ water head, but not 198m. For schemes S-198-PID and S-198FOPID, PTGS can obtain good control performance at $205 \mathrm{~m}$ and $210 \mathrm{~m}$ working water heads, which conforms to the actual physical phenomenon that the control parameters suitable for low water head may also suitable for middle or high water heads. However, due to the lack of comprehensive consideration for complex working conditions, the adaptability and robustness of the PID and FOPID controllers optimized 


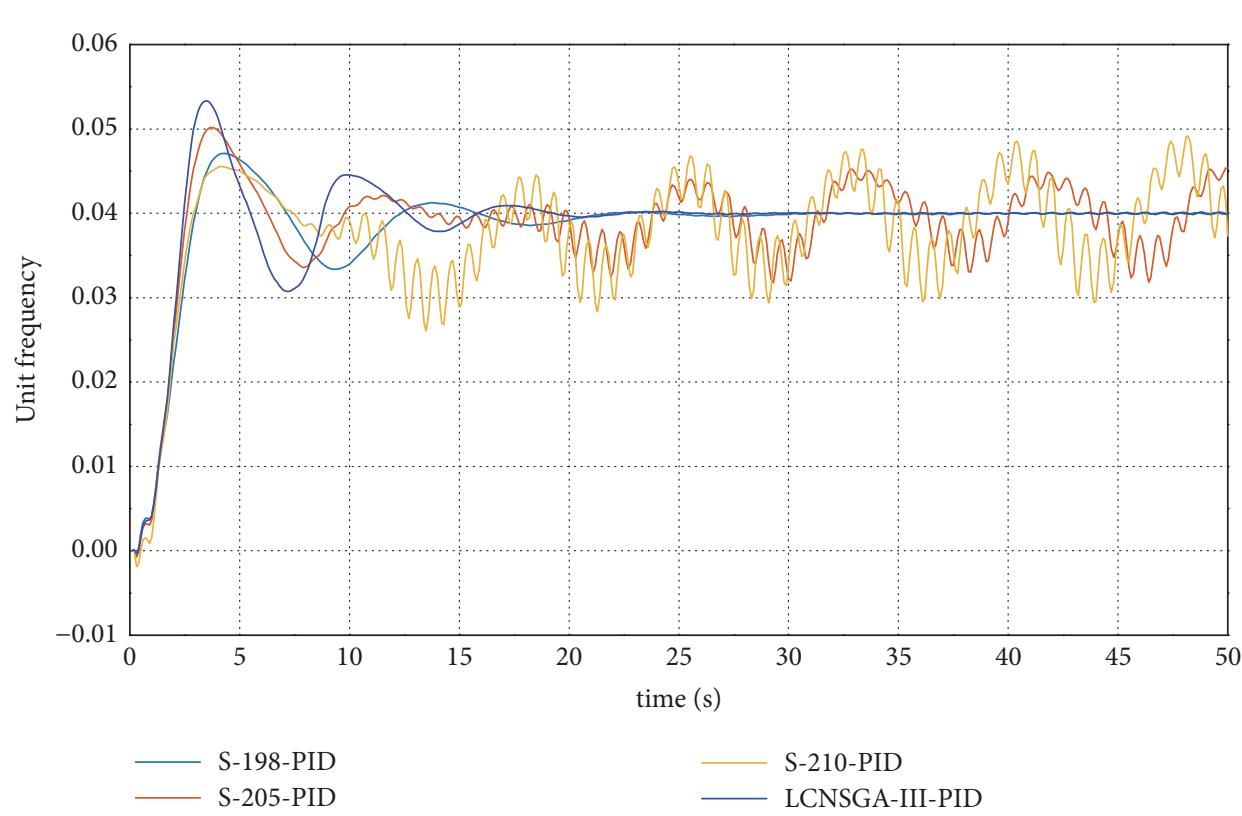

(a) PID controller

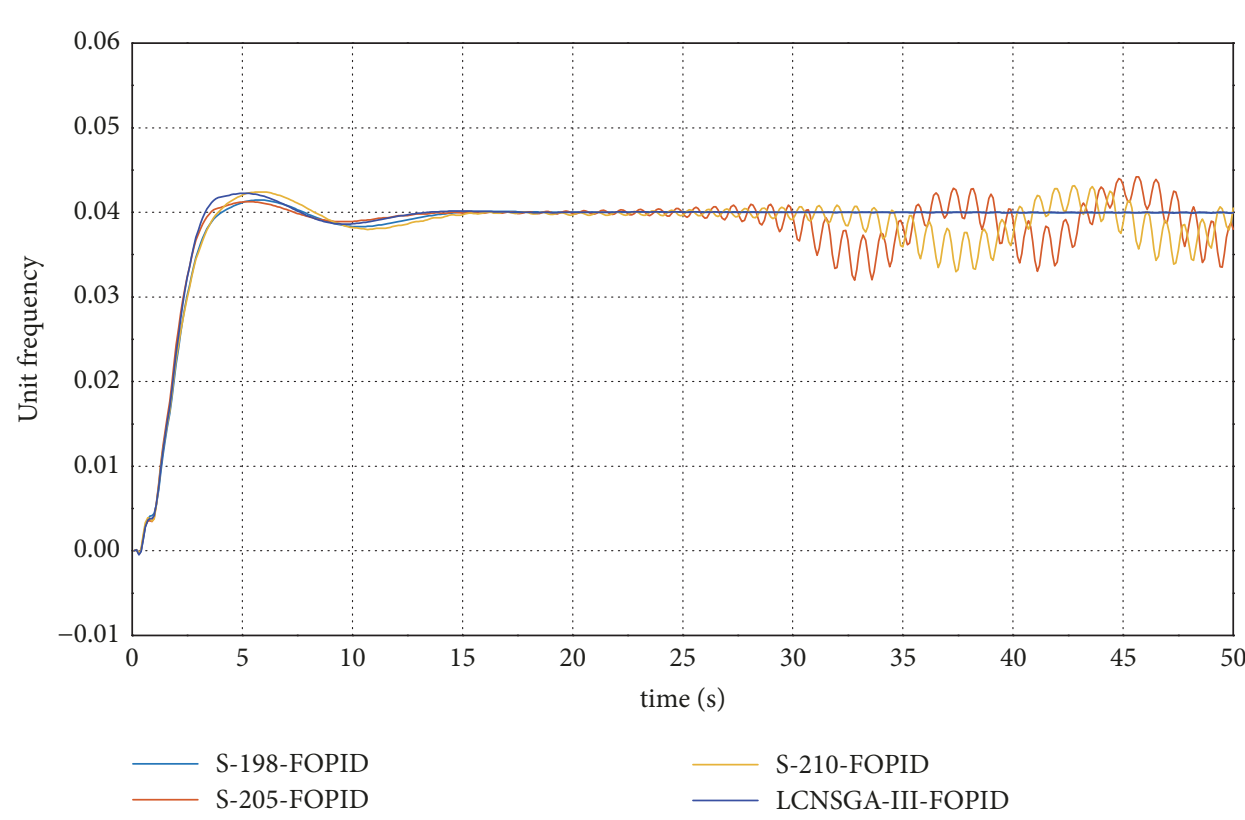

(b) FOPID controller
S-198-PID

ITAE: 5.68 ITSE: 0.04

Stable time (s): 49.9

Overshoot (\%): 17.7

S-205-PID

ITAE: 37.28 ITSE:0.17

Stable time (s): /

Overshoot (\%): 25.4

S-210-PID

ITAE: 54.29 ITSE: 0.35

Stable time (s): /

Overshoot (\%): 22.8

LCNSGA-III-PID

ITAE: 5.43 ITSE: 0.04

Stable time (s): /

Overshoot (\%): 33.3

FIGURE 7: Unit frequency obtained at $198 \mathrm{~m}$ working water head.

at complex operating conditions simultaneously using multiobjective optimization algorithms should be studied and investigated.

5.2.4. Analysis of Controllers Optimized under Multiworking Conditions. The Pareto fronts obtained by LCNSGA-IIIPID and LCNSGA-III-FOPID are shown in Figure 10. From Figure 10, it can be noticed that the Pareto front of LCNSGAIII-FOPID can dominate that of LCNSGA-III-PID, which further demonstrate the superiority of the FOPID controller. It can also be noticed from Figure 10 that the $\mathrm{ITAE}_{1}$ for low water head $(198 \mathrm{~m})$ and the $\mathrm{ITAE}_{2}$ for high water head $(210 \mathrm{~m})$ are two conflicting indices. The optimal solution for working condition at low water head is not the best one for working condition at high water head. The employment of multiobjective optimization algorithms to optimize the two objectives simultaneously can help researchers find the compromise optimal solutions. Compare the ITAE indices for S-198-PID and S-210-PID in Table 5 with the Pareto front obtained by LCNSGA-III-PID; in Figure 10, it can be found that the ITAE indices for the two schemes are the nearest to the two edge solutions of the Pareto front of 


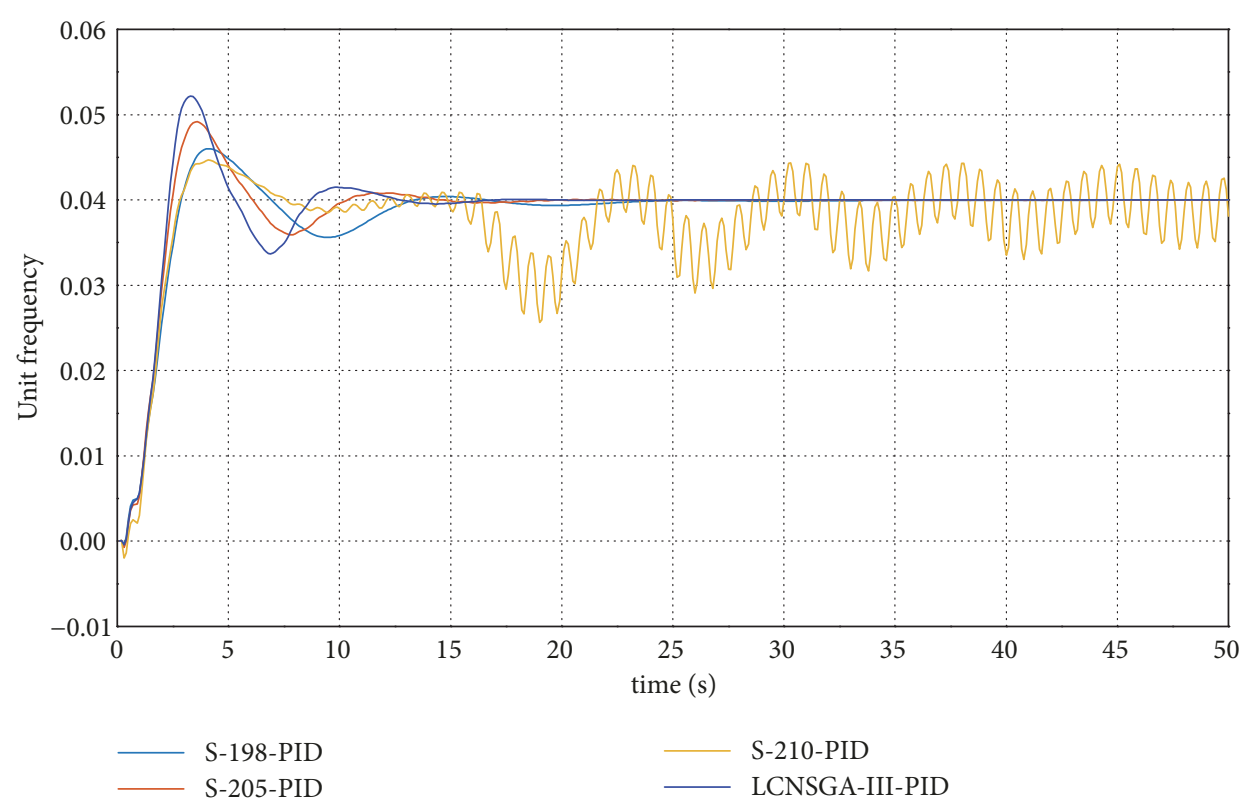

(a) PID controller

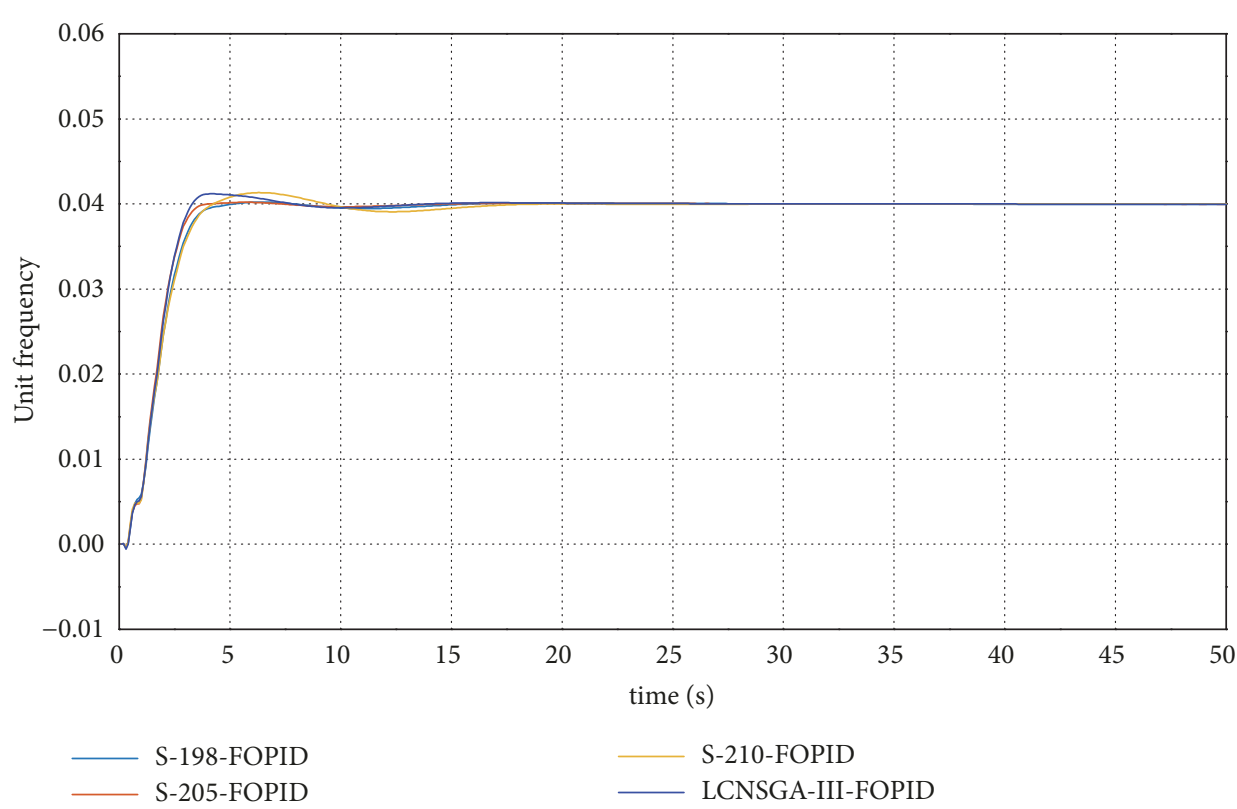

(b) FOPID controller
S-198-PID

ITAE: 4.10 ITSE: 0.02

Stable time (s): 35.6

Overshoot (\%): 15.0

S-205-PID

ITAE: 2.69 ITSE: 0.02

Stable time (s): 29.3

Overshoot (\%): 22.9

S-210-PID

ITAE: 37.88 ITSE: 0.21

Stable time (s): /

Overshoot (\%): 11.7

LCNSGA-III-PID

ITAE: 1.37 ITSE: 0.02

Stable time (s): 23.3

Overshoot (\%): 2.3

FIGURE 8: Unit frequency obtained at $205 \mathrm{~m}$ working water head.

LCNSGA-III-PID. Similar phenomenon also exists between the solutions obtained by S-198-FOPID and S-210-FOPID and the Pareto optimal solutions obtained by LCNSGA-IIIFOPID, which demonstrates that the pursing for a single objective (high or low water head) is at the cost of the other objective (corresponding low or high water head). What is more, compared with the schemes for single working conditions, the adaptability and robustness of the controllers can be greatly improved using multiobjective optimization schemes. A set of Pareto optimal solutions are obtained using multiobjective optimization schemes; it is convenient for the operator to select the most appropriate control parameters when the working condition changes or much more attention should be paid to the extremely low water head or high water head working conditions.

The effectiveness of the developed LCNSGA-III algorithm has been verified using the eight test functions which have been described in Section 5.1. In what follows the superiority of the developed LCNSGA-III algorithm is further demonstrated by applying it to PTGS. The Pareto fronts of PID and FOPID controllers optimized by the two algorithms have been illustrated in Figures 11(a) and 11(b), respectively. 


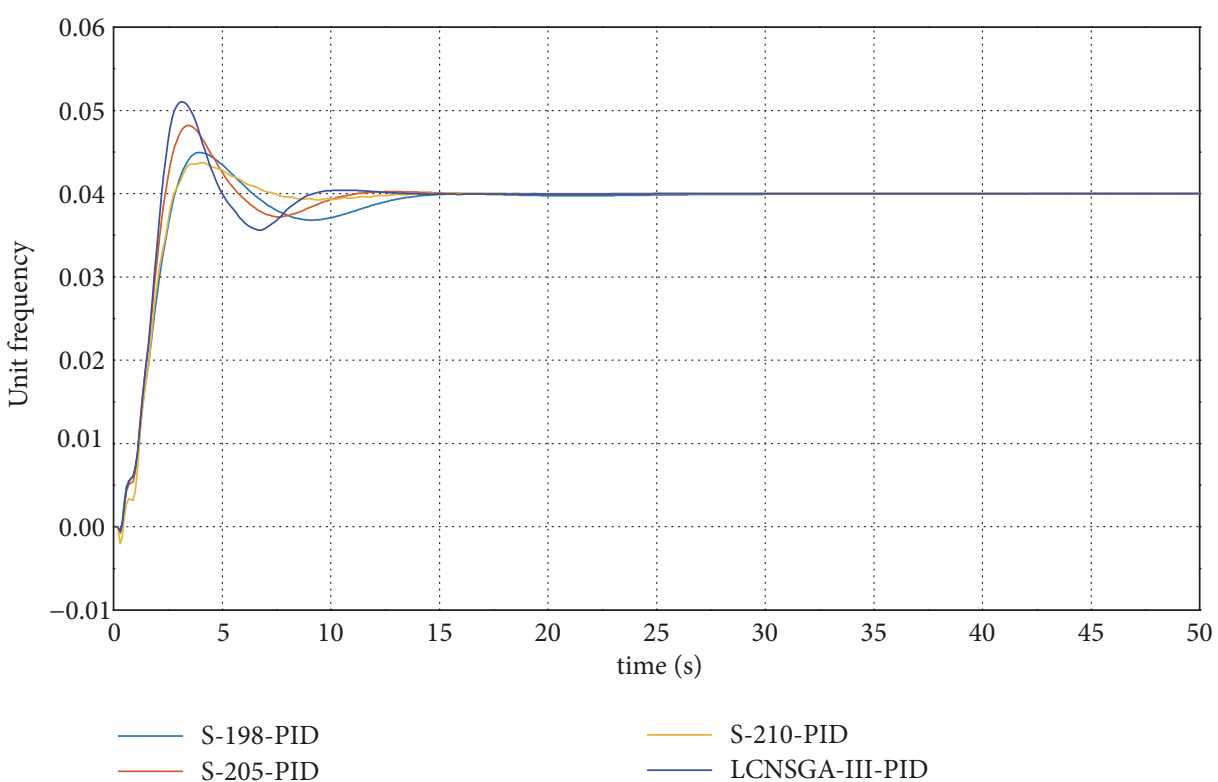

(a) PID controller

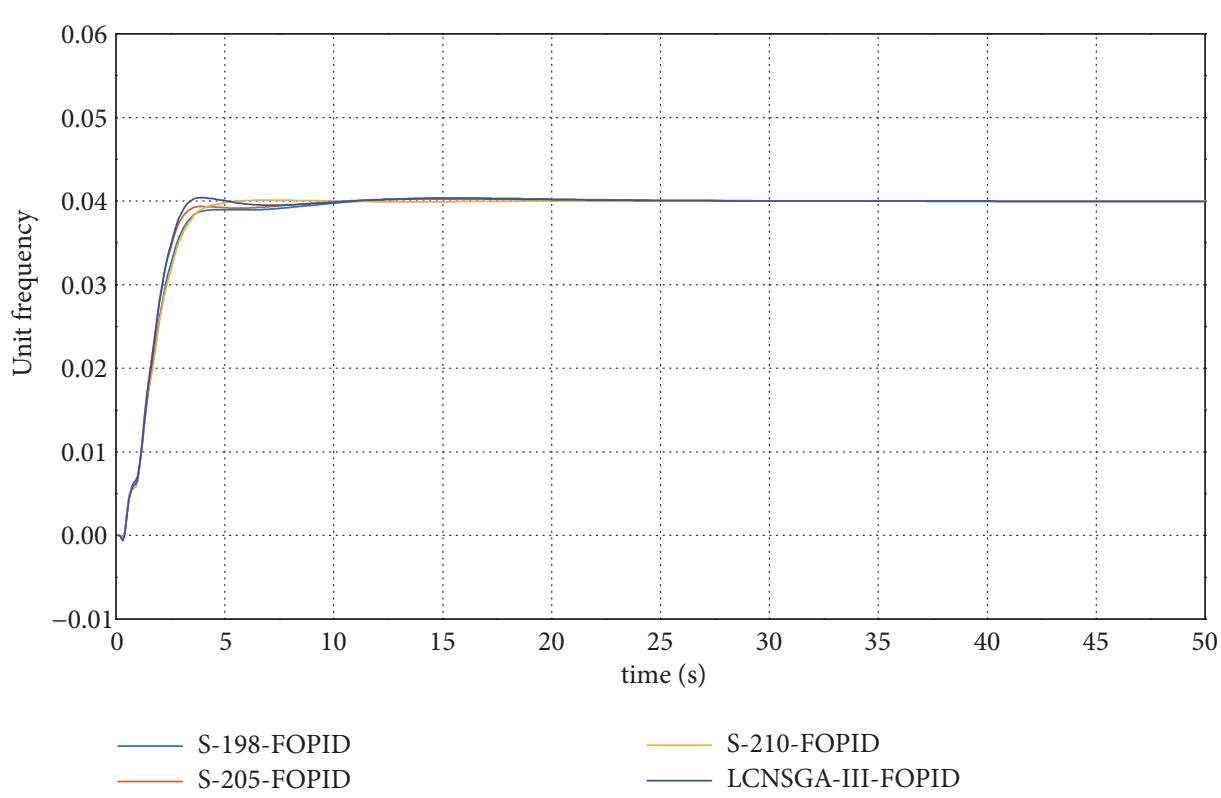

(b) FOPID controller
S-198-PID

ITAE: 3.16 ITSE: 0.02

Stable time (s): 35.5

Overshoot (\%): 12.4

S-205-PID

ITAE: 1.93 ITSE: 0.02

Stable time (s): 20.1

Overshoot (\%): 20.5

\section{S-210-PID}

ITAE: 1.34 ITSE: 0.02

Stable time (s): 18.6

Overshoot (\%): 9.3

LCNSGA-III-PID

ITAE: 1.37 ITSE: 0.02

Stable time (s): 23.3

Overshoot (\%): 2.3

FIGURE 9: Unit frequency obtained at 210m working water head.

It can be observed from Figure 11 that the Pareto optimal solutions obtained by LCNSGA-III can dominate almost all of those obtained by NSGA-III. And the Pareto front obtained by LCNSGA-III distributes more uniformly and extensively. It can be found in Table 5 that the performances indices for the compromise Pareto optimal solution of LCNSGA-III are all smaller than NSGA-III, which further demonstrates the superiority of LCNSGA-III in optimizing the MOFOPID problem. For example, the improved percentages of scheme LCNSGA-III-PID to scheme NSGA-III-PID are $62.9 \%, 50.0 \%$, and $84.9 \%$ in terms of ITAE, ITSE, and OSO, respectively in the training stage $(198 \mathrm{~m})$. The improved percentages in the testing stage $(205 \mathrm{~m})$ are $46.1 \%, 33.3 \%$, and $91.3 \%$ in terms of ITAE, ITSE, and OSO, respectively.

\section{Conclusions}

In order to make PSUs adaptable to the changes of working environment and improve their control quality and stability, this study constructs a multiobjective optimization framework to optimize the FOPID controller for PTGS under multiworking conditions. An LCNSGA-III algorithm based on Latin-hypercube sampling and chaos theory is proposed to solve the MO-FOPID problem under multiworking 


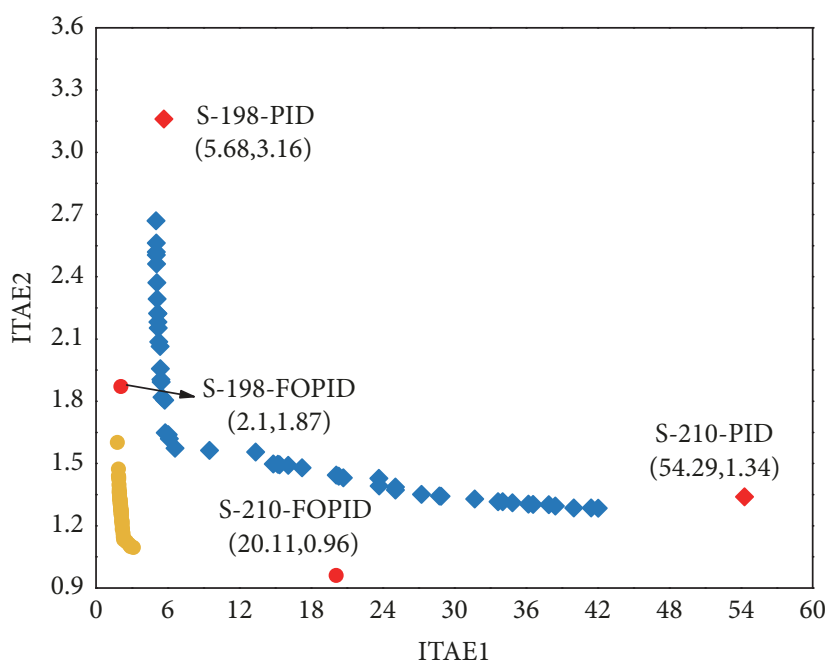

$\rightarrow$ LCNSGA-III-PID

- LCNSGA-III-FOPID

FigURE 10: Pareto fronts obtained by LCNSGA-III-PID, LCNSGA-III-FOPID, and their comparison with single-objective solutions.

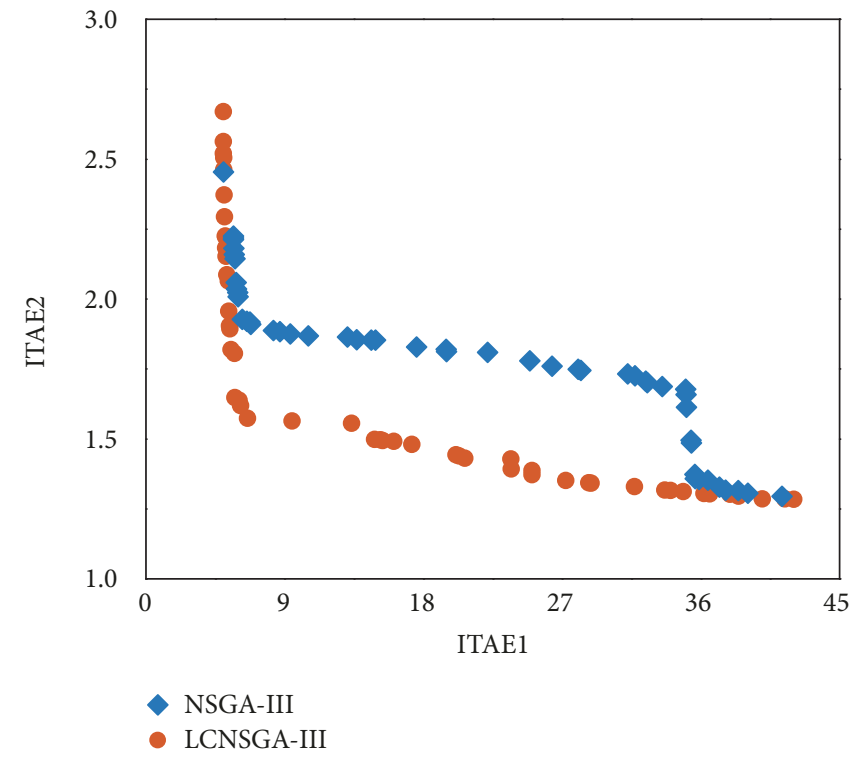

(a) PID

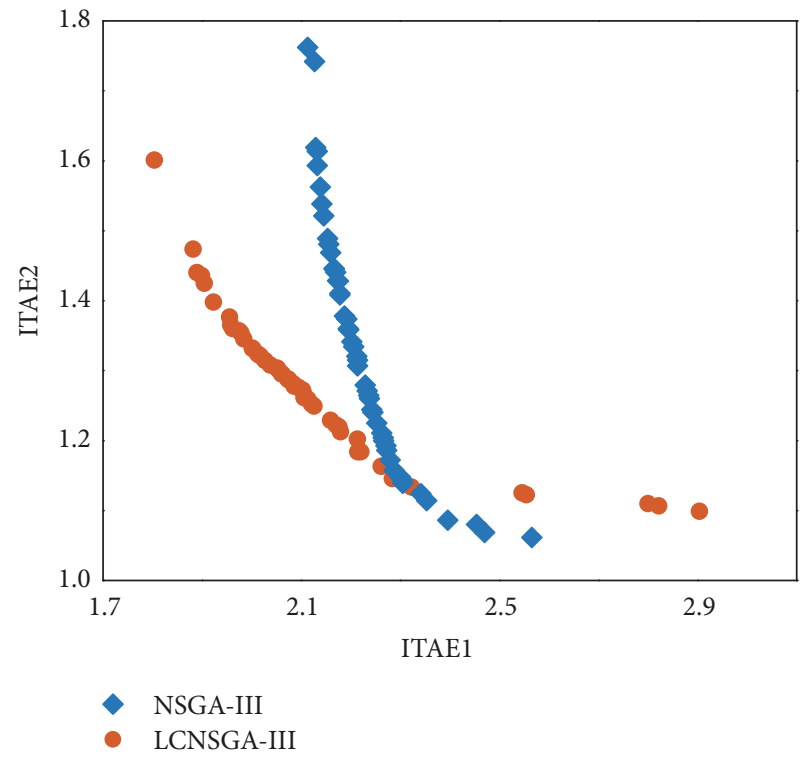

(b) FOPID

FIGURE 11: Comparison of Pareto fronts obtained by LCNSGA-III and NSGA-III for PID and FOPID controllers.

conditions. Implementation of the MO-FOPID controller optimized by LCNSGA-III relies on the simultaneous optimization of two complementary features: the ITAE index under low and high water heads. The experiment results indicate the following:

(1) The classical NSGA-III algorithm is improved using the Latin hypercube sampling- based initialization technique and the chaotic crossover and mutation operators. Experiments of the eight test functions show that the improvement strategies can effectively improve the convergence and diversity of the Pareto optimal front of the NSGA-III algorithm.
(2) Compared with the traditional PID controller, the FOPID can effectively suppress the frequency oscillation of PSUs in the "S" characteristic area running at middle or low working water heads and can enhance the dynamic response performance of PTGS.

(3) This study extends the single-objective optimization under single working conditions to the multiobjective optimization framework under multiworking conditions. The multiobjective framework has provided better dynamic performances than the single-objective optimization methods. The multiobjective implementation of FOPID makes it much more convenient for a operator to select the most 
appropriate control parameters for a certain working condition.

This work sets a basis for research on multiobjective optimization of a FOPID controller of PTGS under multiworking conditions. The extension of the single-objective optimization under a single working-condition to the multiobjective framework under complex working conditions can provide new control law and optimization algorithms for the optimal control of PTGS. What is more, the proposed LCNSGA-III algorithm can be easily extended to optimization problems in other fields of scientific research and industrial application.

\section{Data Availability}

The data used to support the findings of this study are available from the corresponding author upon request.

\section{Conflicts of Interest}

The authors declare no conflicts of interest.

\section{Acknowledgments}

This work is supported by the National Natural Science Foundation of China (No. 51741907), the National Natural Science Foundation of China (NSFC) (No. 51709121), and the National Natural Science Foundation of China (No. 51709122).

\section{References}

[1] R. N. Allan, R. Li, and M. M. Elkateb, "Modelling of pumpedstorage generation in sequential Monte Carlo production simulation," Generation, Transmission and Distribution, IEE Proceedings, vol. 145, no. 5, pp. 611-615, 1998.

[2] W. Wang, C. Li, X. Liao, and H. Qin, "Study on unit commitment problem considering pumped storage and renewable energy via a novel binary artificial sheep algorithm," Applied Energy, vol. 187, pp. 612-626, 2017.

[3] J. Zhou, Z. Zhao, C. Zhang, C. Li, and Y. Xu, "A real-time accurate model and its predictive fuzzy PID controller for pumped storage unit via error compensation," Energies, vol. 11, no. 1, 2018.

[4] J. Zhou, C. Zhang, T. Peng, and Y. Xu, "Parameter identification of pump turbine governing system using an improved backtracking search algorithm," Energies, vol. 11, no. 7, p. 1668, 2018.

[5] C. Zhang, C. Li, T. Peng et al., "Modeling and synchronous optimization of pump turbine governing system using sparse robust least squares support vector machine and hybrid backtracking search algorithm," Energies, vol. 11, no. 11, p. 3108, 2018.

[6] Y. Xu, Y. Zheng, Y. Du, W. Yang, X. Peng, and C. Li, "Adaptive condition predictive-fuzzy PID optimal control of start-up process for pumped storage unit at low head area," Energy Conversion and Management, vol. 177, pp. 592-604, 2018.

[7] K. Natarajan, "Robust PID controller design for hydroturbines," IEEE Transactions on Energy Conversion, vol. 20, no. 3, pp. 661667, 2005.

[8] R.-E. Precup, A.-D. Balint, M.-B. Radac, and E. M. Petriu, "Backtracking search optimization algorithm-based approach to PID controller tuning for torque motor systems," in Proceedings of the 2015 Annual IEEE Systems Conference, pp. 127-132, IEEE, Vancouver, BC, Canada, 2015.

[9] R.-E. Precup, A.-D. Balint, E. M. Petriu, M.-B. Radac, and E.-I. Voisan, "PI and PID controller tuning for an automotive application using backtracking search optimization algorithms," in Proceedings of the IEEE Jubilee International Symposium on Applied Computational Intelligence and Informatics, pp. 161-166, Timisoara, Romania, May 2015.

[10] A. Khodabakhshian and R. Hooshmand, "A new PID controller design for automatic generation control of hydro power systems," International Journal of Electrical Power \& Energy Systems, vol. 32, no. 5, pp. 375-382, 2010.

[11] R. Melício, V. M. F. Mendes, and J. P. S. Catalão, "Fractionalorder control and simulation of wind energy systems with PMSG/full-power converter topology," Energy Conversion and Management, vol. 51, no. 6, pp. 1250-1258, 2010.

[12] V. Haji Haji and C. A. Monje, "Fractional order fuzzy-PID control of a combined cycle power plant using Particle Swarm Optimization algorithm with an improved dynamic parameters selection," Applied Soft Computing, vol. 58, pp. 256-264, 2017.

[13] S. Zhang and L. Liu, "Normalized robust FOPID controller regulation based on small gain theorem," Complexity, vol. 2018, Article ID 5690630, 10 pages, 2018.

[14] L. Liu and S. Zhang, "Robust fractional-order PID controller tuning based on bode's optimal loop shaping," Complexity, vol. 2018, Article ID 6570560, p. 14, 2018.

[15] J.-W. Perng, G.-Y. Chen, and Y.-W. Hsu, "FOPID controller optimization based on SIWPSO-RBFNN algorithm for fractionalorder time delay systems," Soft Computing, vol. 21, no. 14, pp. 4005-4018, 2017.

[16] C. Li, N. Zhang, X. Lai, J. Zhou, and Y. Xu, "Design of a fractional-order PID controller for a pumped storage unit using a gravitational search algorithm based on the Cauchy and Gaussian mutation," Information Sciences, vol. 396, pp. 162-181, 2017.

[17] Y. Xu, J. Zhou, Y. Zhang, W. Fu, Y. Zheng, and X. Zhang, "Parameter optimization of robust non-fragile fractional order pid controller for pump turbine governing system," in Proceedings of the in Sixth International Conference on Instrumentation Measurement, Computer, pp. 15-18, 2016.

[18] Y. Xu, J. Zhou, X. Xue, W. Fu, W. Zhu, and C. Li, "An adaptively fast fuzzy fractional order PID control for pumped storage hydro unit using improved gravitational search algorithm," Energy Conversion and Management, vol. 111, pp. 67-78, 2016.

[19] C. Jiang, Y. Ma, and C. Wang, "PID controller parameters optimization of hydro-turbine governing systems using deterministic-chaotic-mutation evolutionary programming (DCMEP)," Energy Conversion and Management, vol. 47, no. 9-10, pp. 1222-1230, 2006.

[20] H. Fang, L. Chen, and Z. Shen, "Application of an improved PSO algorithm to optimal tuning of PID gains for water turbine governor," Energy Conversion and Management, vol. 52, no. 4, pp. 1763-1770, 2011.

[21] P. G. Kou, J. Z. Zhou, H. E. Yao-Yao, X. Q. Xiang, and L. I. Chao-Shun, "Optimal PID governor tuning of hydraulic turbine generators with bacterial foraging particle swarm optimization algorithm," Proceedings of the Csee, vol. 29, no. 26, pp. 101-106, 2009.

[22] Z. Wang, C. Li, X. Lai, N. Zhang, Y. Xu, and J. Hou, "An integrated start-up method for pumped storage units based on 
a novel artificial sheep algorithm," Energies, vol. 11, no. 1, p. 151, 2018.

[23] M. J. Mahmoodabadi and H. Jahanshahi, "Multi-objective optimized fuzzy-PID controllers for fourth order nonlinear systems," Engineering Science and Technology, an International Journal, vol. 19, no. 2, pp. 1084-1098, 2016.

[24] S. Panda, "Multi-objective PID controller tuning for a FACTSbased damping stabilizer using non-dominated sorting genetic algorithm-II," International Journal of Electrical Power \& Energy Systems, vol. 33, no. 7, pp. 1296-1308, 2011.

[25] A.-A. Zamani, S. Tavakoli, and S. Etedali, "Fractional order PID control design for semi-active control of smart baseisolated structures: a multi-objective cuckoo search approach," ISA Transactions, vol. 67, p. 222, 2017.

[26] H. S. Sánchez, F. Padula, A. Visioli, and R. Vilanova, “Tuning rules for robust FOPID controllers based on multi-objective optimization with FOPDT models," ISA Transactions, vol. 66, pp. 344-361, 2017.

[27] S.-Z. Zhao, M. W. Iruthayarajan, S. Baskar, and P. N. Suganthan, "Multi-objective robust PID controller tuning using two lbests multi-objective particle swarm optimization," Information Sciences, vol. 181, no. 16, pp. 3323-3335, 2011.

[28] Z. Chen, X. Yuan, B. Ji, P. Wang, and H. Tian, "Design of a fractional order PID controller for hydraulic turbine regulating system using chaotic non-dominated sorting genetic algorithm II," Energy Conversion and Management, vol. 84, pp. 390-404, 2014.

[29] Z. Chen, Y. Yuan, X. Yuan, Y. Huang, X. Li, and W. Li, "Application of multi-objective controller to optimal tuning of PID gains for a hydraulic turbine regulating system using adaptive grid particle swam optimization," ISA Transactions, vol. 56, pp. 173-187, 2015.

[30] T. Peng, J. Zhou, C. Zhang, and N. Sun, "Modeling and combined application of orthogonal chaotic NSGA-II and Improved TOPSIS to optimize a conceptual hydrological model," Water Resources Management, pp. 1-19, 2018.

[31] D. Xue, C. Zhao, and Y. Chen, "A modified approximation method of fractional order system," in Proceedings of the IEEE International Conference on Mechatronics and Automation, pp. 1043-1048, 2006.

[32] I. Podlubny, "Fractional-order systems and PI $\lambda \mathrm{D} \mu$-controllers," in Proceedings of the IEEE Trans on Automatic Control, vol. 44, 1999.

[33] M. Zamani, M. Karimi-Ghartemani, N. Sadati, and M. Parniani, "Design of a fractional order PID controller for an AVR using particle swarm optimization," Control Engineering Practice, vol. 17, no. 12, pp. 1380-1387, 2009.

[34] C. Li, Y. Mao, J. Zhou, N. Zhang, and X. An, "Design of a fuzzyPID controller for a nonlinear hydraulic turbine governing system by using a novel gravitational search algorithm based on Cauchy mutation and mass weighting," Applied Soft Computing, vol. 52, pp. 290-305, 2017.

[35] Y. Xu, C. Li, Z. Wang, N. Zhang, and B. Peng, "Load frequency control of a novel renewable energy integrated micro-grid containing pumped hydropower energy storage," IEEE Access, vol. 6, pp. 29067-29077, 2018.

[36] H. Jain and K. Deb, "An evolutionary many-objective optimization algorithm using reference-point based nondominated sorting approach, Part II: Handling constraints and extending to an adaptive approach," IEEE Transactions on Evolutionary Computation, vol. 18, no. 4, pp. 602-622, 2014.
[37] K. Deb, S. Agrawal, A. Pratap, and T. Meyarivan, "A fast elitist non-dominated sorting genetic algorithm for multi-objective optimization: NSGA-II," in Proceedings of the International Conference on Parallel Problem Solving From Nature, vol. 1917, pp. 849-858.

[38] S. Poles, Y. Fu, and E. Rigoni, The effect of initial population sampling on the convergence of multi-objective genetic algorithms, Springer, Berlin,German, 2009.

[39] W. Fu, K. Wang, C. Li, X. Li, Y. Li, and H. Zhong, "Vibration trend measurement for a hydropower generator based on optimal variational mode decomposition and an LSSVM improved with chaotic sine cosine algorithm optimization," Measurement Science and Technology, vol. 30, no. 1, p. 015012, 2019.

[40] H. Lu, R. Niu, J. Liu, and Z. Zhu, "A chaotic non-dominated sorting genetic algorithm for the multi-objective automatic test task scheduling problem," Applied Soft Computing, vol. 13, no. 5, pp. 2790-2802, 2013.

[41] T. Habutsu, Y. Nishio, I. Sasase, and S. Mori, "A secret key cryptosystem by iterating a chaotic map," in International Conference on Theory and Application of Cryptographic Techniques, pp. 127$140,1991$.

[42] K. Deb, A. Pratap, S. Agarwal, and T. Meyarivan, "A fast and elitist multiobjective genetic algorithm: NSGA-II," IEEE Transactions on Evolutionary Computation, vol. 6, no. 2, pp. 182197, 2002.

[43] K. Deb, L. Thiele, M. Laumanns, and E. Zitzler, "Scalable multiobjective optimization test problems," in Proceedings of the Congress on Evolutionary Computation (CEC '02), pp. 825-830, May 2002.

[44] X. Lai, C. Li, N. Zhang, and J. Zhou, "A multi-objective artificial sheep algorithm," Neural Computing and Applications, 2018.

[45] K. Deb and H. Jain, "An evolutionary many-objective optimization algorithm using reference-point- based nondominated sorting approach, part I: solving problems with box constraints," IEEE Transactions on Evolutionary Computation, vol. 18, no. 4, pp. 577-601, 2014.

[46] K. Deb, Multiobjective Optimization Using Evolutionary Algorithms, New York, NY, USA, Wiley, 2001.

[47] C. Zhang, J. Zhou, C. Li, W. Fu, and T. Peng, "A compound structure of ELM based on feature selection and parameter optimization using hybrid backtracking search algorithm for wind speed forecasting," Energy Conversion and Management, vol. 143, pp. 360-376, 2017. 


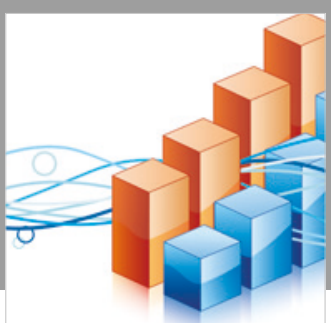

Advances in

Operations Research

\section{-n-m}
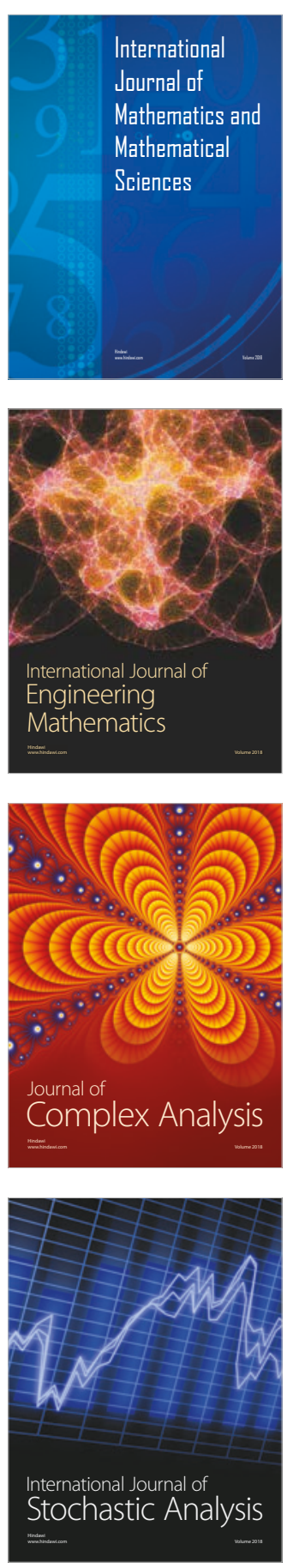
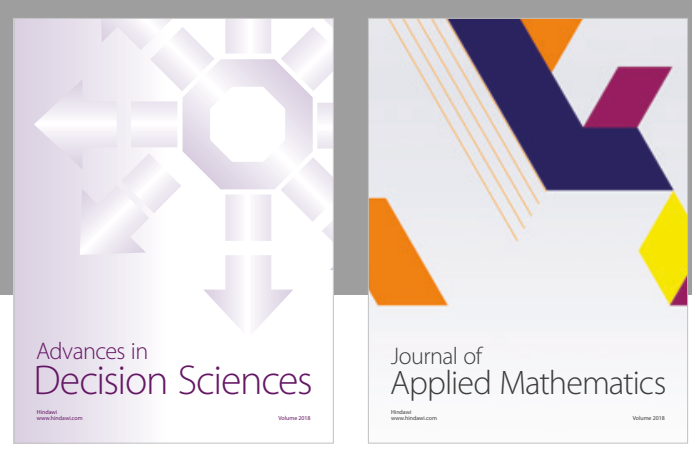

Journal of

Applied Mathematics
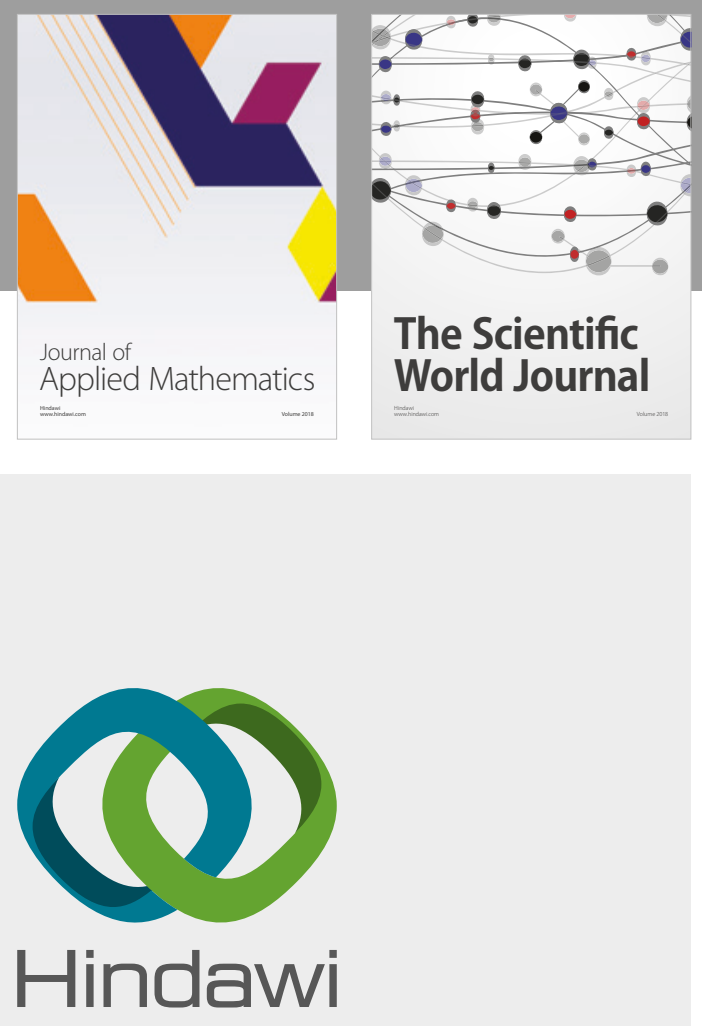

Submit your manuscripts at

www.hindawi.com

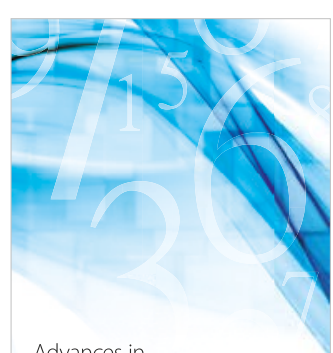

Advances in
Numerical Analysis
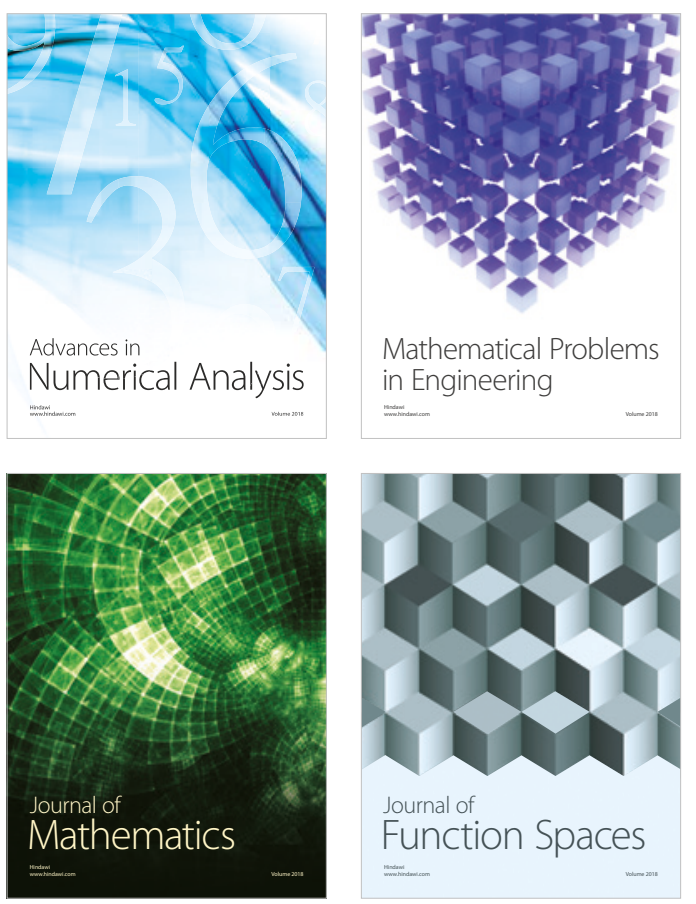

Mathematical Problems in Engineering

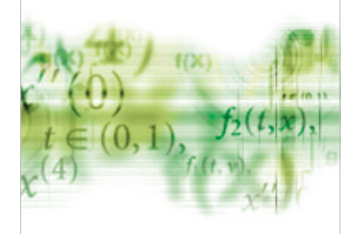

International Journal of

Differential Equations

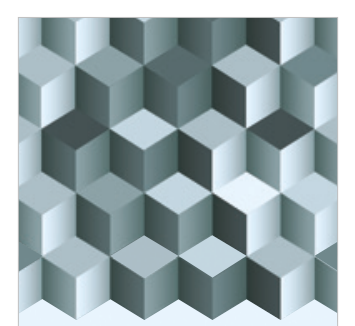

Journal of

Function Spaces
The Scientific

World Journal

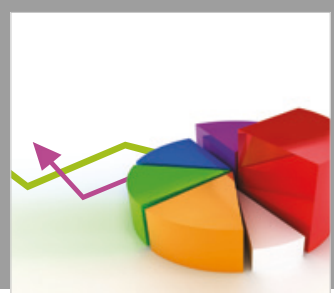

Journal of

Probability and Statistics
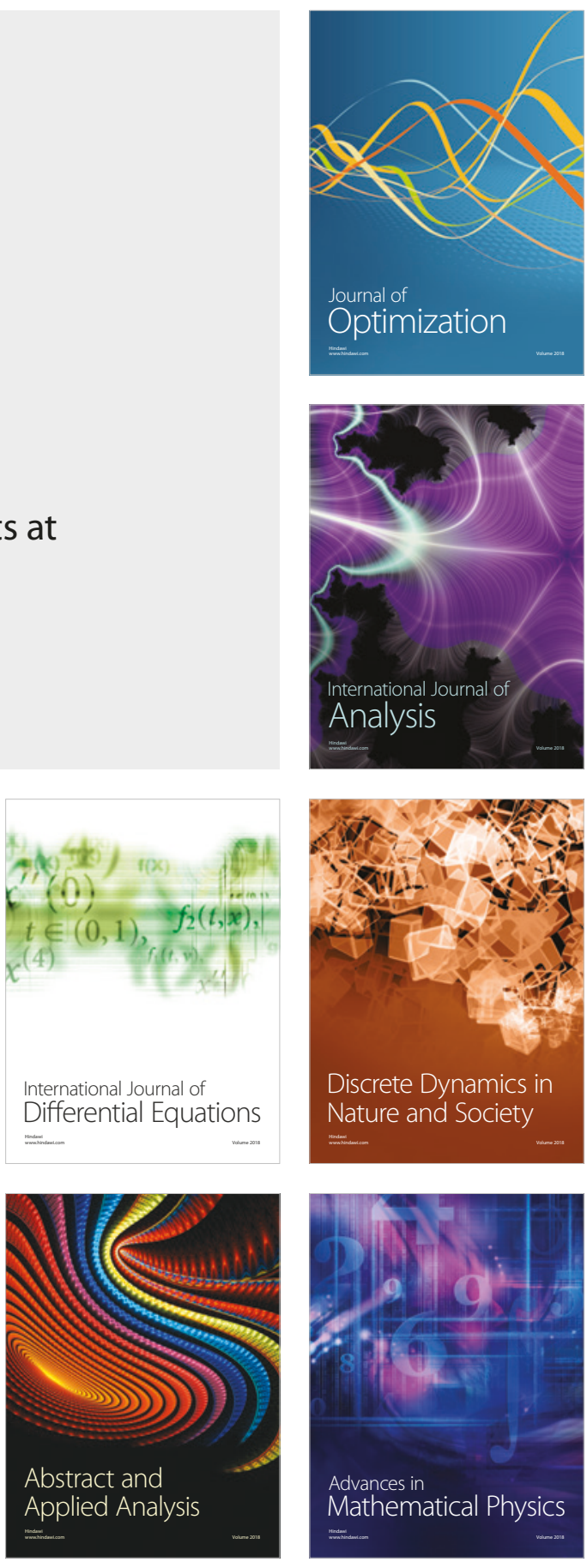